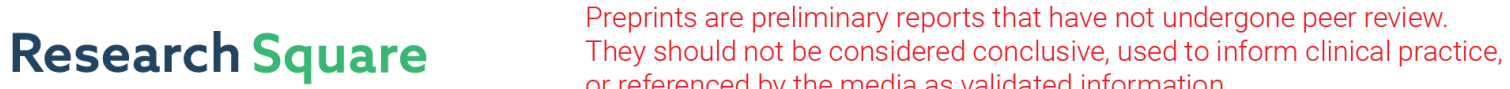 or referenced by the media as validated information. \\ Soliton Molecules, Rational Positon Solution and Rogue Waves for the Extended Complex Modified KdV Equation
}

\section{Lin Huang}

Hangzhou Dianzi University

Nannan Lv ( $\triangle$ Ivkenan1122@163.com)

Hangzhou Dianzi University School of Science

\section{Research Article}

Keywords: Darboux transformation, Soliton molecule, Positon solution, Rational positon solution, Rogue waves solution

Posted Date: May 11th, 2021

DOI: https://doi.org/10.21203/rs.3.rs-434604/v1

License: (c) (1) This work is licensed under a Creative Commons Attribution 4.0 International License.

Read Full License

Version of Record: A version of this preprint was published at Nonlinear Dynamics on August 5th, 2021. See the published version at https://doi.org/10.1007/s11071-021-06764-x. 


\title{
Soliton molecules, rational positons and rogue waves for the extended complex modified KdV equation
}

\author{
Lin Huang *and Nannan $\mathrm{Lv}^{\dagger}$ \\ School of Science, Hangzhou Dianzi University, 310018 Zhejiang, China.
}

May 6, 2021

\begin{abstract}
We consider the integrable extended complex modified Korteweg-de Vries equation, which is generalized modified $\mathrm{KdV}$ equation. The first part of the article considers the construction of solutions via the Darboux transformation. We obtain some exact solutions, such as soliton molecules, positon solutions, rational positon solutions, and rogue waves solutions. The second part of the article analyzes the dynamics of rogue waves. By means of the numerical analysis, under the standard decomposition, we divide the rogue waves into three patterns: fundamental pattern, triangular pattern and ring pattern. For the fundamental pattern, we define the length and width of the rogue waves and discuss the effect of different parameters on rogue waves.
\end{abstract}

Keywords: Darboux transformation, Soliton molecule, Positon solution, Rational positon solution, Rogue waves solution.

\section{Introduction}

It is well-known that the integrable partial differential equations(PDEs) are integral part of modern mathematical and theoretical physics with far-reaching implications from pure mathematics to the applied sciences. Integrable equations have many useful properties: such as Lax pairs, Hamilton structure, conservation laws, exact solutions, and so on. Many phenomena in science can be described by integrable equations, so for an integrable equation, it is important to find their exact solutions. At present, there are many methods to find the exact solution of the equation, such as Lie group [4], the Darboux transformation (DT) [10,24, 28], the Hirota bilinear method [11], Bäcklund transformation [23], algebraic geometry method [3], and the famous inverse scattering transformation $[1,2]$, etc.

The Darboux transformation is a powerful method to construct solutions for integrable PDEs. In particular, the well-known soliton solution appearing in many physical motivated PDEs like the NLS equation, complex modified KdV equation can be computed thereby. In 2012, Ling, Guo et al obtain that the so-called rogue waves solutions of the nonlinear Schrödinger equation by the generalized Darboux transformation [7]. The concept of rogue waves originated from oceanography [15]. Oceanic rogue waves are surface gravity waves which height is much larger than expected for the sea state. The common operational definition requires them to be at least twice as large as the significant wave height. At first, people did not understand the mechanism of this phenomenon, but with the development of science and technology, some ocean probes observed this phenomenon. For example, the shape of large surface waves on the open sea and the Draupner new year wave [29]. Now rogue waves have been proposed in many fields, such as

\footnotetext{
*lin.huang@hdu.edu.cn

†Corresponding author: lvkenan1122@163.com
} 
Nonlinear optics [27], Finance [32], Bose-Einstein condensates [12], plasmas [25], etc. For the more research on rogue waves, please refer to monograph [9] and its references.

Recently, so-called soliton molecules were obtained in optical experiments and attracted people's attention. Whereafter, the scientists have discovered soliton molecules in Bose-Einstein condensates [21], Few-cycle mode-locked laser [13], etc. In 2020, Lou presented a velocity resonance mechanism and theoretically obtained soliton molecules of integrable systems and asymmetric solitons three-dimensional fluid system [18].

The classical complex modified Korteweg-de Vries (cmKdV) equations can be written as

$$
q_{t}+\alpha\left(q_{x x x}+6|q|^{2} q_{x}\right)=0
$$

where $q=q(t, x)$ is a complex function. The cmKdV equation has many applications in science. For example, the cmKdV equation has been proposed as a model for nonlinear evolution of plasma waves [16], it has been derived to describe the propagation of transverse waves in a molecular chain model [8] and in a generalized elastic solid [5,6]. In [14], He et al constructed a generalized Darboux transformation for the cmKdV equation which obtain the rogue waves solution and analyze the dynamic of rogue waves. The soliton molecules for the cmKdV equation considered in [33].

In this paper, we investigate an extended complex modified Korteweg-de Vries (ecmKdV) equation, which takes the form

$\left.q_{t}+\alpha\left(q_{x x x}+6|q|^{2} q_{x}\right)+\beta\left[-30|q|^{4} q_{x}-10 q_{x}^{2} q_{x}^{*}-20 q^{*} q_{x} q_{x x}-10 q\left(q_{x}^{*} q_{x x}+q_{x} q_{x x}^{*}+q^{*} q_{x x x}\right)-q_{x x x x x}\right)\right]=0$,

where $\alpha \ll 1$ and $\beta \ll 1$ stand for the third-order and fifth-order dispersion coefficients matching with the relevant nonlinear terms, respectively. If we take the $\beta=0$, the Eq.(2) reduce to cmKdV equation (1). If we use $-\beta$ instead of $\beta$ and take the $q(x, t)$ is real function in ecmKdV equation $(2)$, the ecmKdV equation can be reduced to the following equation

$$
q_{t}+\alpha\left(q_{x x x}+6 q^{2} q_{x}\right)+\beta\left(30 q^{4} q_{x}+10 q_{x}^{3}+40 q q_{x} q_{x x}+10 q^{2} q_{x x x}+q_{x x x x x}\right)=0 .
$$

Wazwaz and $\mathrm{Xu}[30]$ have considered the Painlevé test and multi-soliton solutions via the simplified Hirota'direct method for Eq.(3). The conservation laws, Darboux transformation and periodic solutions obtained in [31]. The soliton molecules of the Eq.(3) are obtained in [26]. The long time asymptotic for the equation (3) with initial data or initial-boundary values are considered in $[19,20]$. In [17], the authors have obtained the explicit solitons and breather solutions for the equation (3) by the Riemann-Hilbert method. Our aim is to construct the exact solutions for Eq.(2) through the Darboux transformation technique in this paper.

Our manuscript is organized as follows: In Section 2, we introduce the Lax pair and the Darboux transformation for Eq.(2). In Section 3, we obtain soliton molecules, positon solutions of Eq.(2) from seed solution $q=0$. In Section 4, we obtain rational positon solutions of Eq.(2) from nonzero seed solution $q=c$. In particular, we construct the higher order rogue waves solutions from a periodic seed with constant amplitude and analyze their structures in detail by choosing suitable system parameters in Section 5. We give the conclusions in Section 6 .

\section{Lax pair and Darboux transformation}

\subsection{Lax pair}

Introducing $r=q^{*}$, Eq.(2) can be rewrite as follows,

$q_{t}+\alpha\left(q_{x x x}+6 q r q_{x}\right)+\beta\left[-30 q^{2} r^{2} q_{x}-10 q_{x}^{2} r_{x}-20 r q_{x} q_{x x}-10 q\left(r_{x} q_{x x}+q_{x} r_{x x}+r q_{x x x}\right)-q_{x x x x x}\right]=0$.

According to the AKNS method [1], we obtain the Lax pair corresponding to Eq.(2),

$$
\psi_{x}=M \psi, \quad \psi_{t}=W \psi
$$


where

$$
\begin{gathered}
W=W_{5} \lambda^{5}+W_{4} \lambda^{4}+W_{3} \lambda^{3}+W_{2} \lambda^{2}+W_{1} \lambda+W_{0}, \\
\psi=\left(\begin{array}{c}
\phi_{1} \\
\phi_{2}
\end{array}\right), M=\left(\begin{array}{cc}
-\lambda & q \\
-r & \lambda
\end{array}\right), W_{5}=\left(\begin{array}{cc}
-16 \beta & 0 \\
0 & 16 \beta
\end{array}\right), W_{4}=\left(\begin{array}{cc}
0 & 16 \beta q \\
-16 \beta r & 0
\end{array}\right), W_{0}=\left(\begin{array}{cc}
w_{11} & w_{12} \\
w_{21} & w_{22}
\end{array}\right), \\
W_{3}=\left(\begin{array}{cc}
4 \alpha-8 \beta q r & -8 \beta q_{x} \\
-8 \beta r_{x} & -4 \alpha+8 \beta q r
\end{array}\right), W_{2}=\left(\begin{array}{cc}
\beta\left(4 r q_{x}-4 q r_{x}\right) & -4 \alpha q-4 \beta\left(-2 q^{2} r-q_{x x}\right) \\
4 \alpha r+4 \beta\left(-2 q r^{2}-r_{x x}\right) & \beta\left(4 q r_{x}-4 r q_{x}\right)
\end{array}\right), \\
W_{1}=\left(\begin{array}{cc}
2 \alpha q r+\beta\left(-6 q^{2} r^{2}+2 q_{x} r_{x}-2 r q_{x x}-2 q r_{x x}\right) & 2 \alpha q_{x}-2 \beta\left(6 q r q_{x}+q_{x x x}\right) \\
2 \alpha r_{x}-2 \beta\left(6 q r r_{x}+r_{x x x}\right) & -2 \alpha q r-\beta\left(-6 q^{2} r^{2}+2 q_{x} r_{x}-2 r q_{x x}-2 q r_{x x}\right)
\end{array}\right), \\
w_{11}=-\alpha\left(r q_{x}-q r_{x}\right)+\beta\left[6 q r^{2} q_{x}-r_{x}\left(6 q^{2} r+q_{x x}\right)+q_{x} r_{x x}+r q_{x x x}-q r_{x x x}\right], \\
w_{12}=-2 \alpha q^{2} r-\alpha q_{x x}+\beta\left(6 q^{3} r^{2}+6 r q_{x}^{2}+4 q q_{x} r_{x}+8 q r q_{x x}+2 q^{2} r_{x x}+q_{x x x x}\right), \\
w_{21}=2 \alpha q r^{2}+\alpha r_{x x}-\beta\left(6 q^{2} r^{3}+6 q r_{x}^{2}+4 r q_{x} r_{x}+8 q r r_{x x}+2 r^{2} q_{x x}+r_{x x x x}\right), \\
w_{22}=\alpha\left(r q_{x}-q r_{x}\right)-\beta\left[6 q r^{2} q_{x}-r_{x}\left(6 q^{2} r+q_{x x}\right)+q_{x} r_{x x}+r q_{x x x}-q r_{x x x}\right] .
\end{gathered}
$$

Taking $r=q^{*}$, if we consider the compatibility condition $M_{t}-W_{x}+[M, W]=0$, one can yield ecmKdV equation (2).

\subsection{Darboux transformation}

In order to obtain the exact solutions of the ecmKdV equation, we will construct the $n$-fold Darboux transformation. First, we consider onefold Darboux transformation $\psi^{[1]}=T_{1} \psi$,

$$
\psi_{x}^{[1]}=M^{[1]} \psi^{[1]}, \quad \psi_{t}^{[1]}=W^{[1]} \psi^{[1]},
$$

$M^{[1]}, W^{[1]}$ and $M, W$ have the same structure except that $q$ and $r$ are converted to $q^{[1]}$ and $r^{[1]}$. We assume the oneflod Darboux transformation

$$
T_{1}=T_{1}(\lambda)=\left(\begin{array}{ll}
a_{1} & b_{1} \\
c_{1} & d_{1}
\end{array}\right) \lambda+\left(\begin{array}{cc}
a_{0} & b_{0} \\
c_{0} & d_{0}
\end{array}\right)
$$

where $a_{i}, b_{i}, c_{i}, d_{i}, i=0,1$ are functions of $x, t$. Combining Eq.(4) and Eq.(5), it's easy to get

$$
T_{x}+T M=M^{[1]} T, \quad T_{t}+T W=W^{[1]} T .
$$

Substituting Eq.(6) into Eq.(7) and comparing the coefficient of $\lambda_{j}, j=0,1,2,3,4,5$, we obtain $b_{1}=c_{1}=0, a_{1, x}=a_{1, t}=d_{1, x}=d_{1, t}=0$,

$$
q^{[1]} d_{1}=q a_{1}+2 b_{0}, \quad r^{[1]} a_{1}=r d_{1}+2 c_{0}
$$

Therefore, $a_{1}$ and $c_{1}$ are constants and we take their value as 1 for the convenience of calculation.

It is not difficult to find that if the eigenfunction $\psi_{j}$ corresponding to the eigenvalue $\lambda_{j}$ satisfies Eq.(4), then the eigenfunction $\psi_{j}^{\prime}$ corresponding to $-\lambda_{j}^{*}$ satisfies Eq.(4). Here

$$
\begin{gathered}
\psi_{j}=\left(\begin{array}{c}
\phi_{j 1} \\
\phi_{j 2}
\end{array}\right), \quad \phi_{j 1}=\phi_{1}\left(x, t, \lambda_{j}\right), \quad \phi_{j 2}=\phi_{2}\left(x, t, \lambda_{j}\right), \\
\psi_{j}^{\prime}=\left(\begin{array}{c}
\phi_{j 2}^{*} \\
-\phi_{j 1}^{*}
\end{array}\right), \quad \phi_{j 2}^{*}=\phi_{2}\left(x, t, \lambda_{j}^{*}\right), \quad \phi_{j 1}^{*}=\phi_{1}\left(x, t, \lambda_{j}^{*}\right) .
\end{gathered}
$$

In the following discussion and calculations, set

$$
\lambda_{2 j}=-\lambda_{2 j-1}^{*}, \quad \phi_{2 j, 1}=\phi_{2 j-1,2}^{*}\left(\lambda_{2 j-1}\right), \quad \phi_{2 j, 2}=-\phi_{2 j-1,1}^{*}\left(\lambda_{2 j-1}\right),(j=1,2, \ldots, n) .
$$

For the onefold DT,

$$
T_{1}=T_{1}(\lambda)=\left(\begin{array}{ll}
1 & 0 \\
0 & 1
\end{array}\right) \lambda+\left(\begin{array}{ll}
a_{0} & b_{0} \\
c_{0} & d_{0}
\end{array}\right)
$$

by using the property of Darboux transformation, i.e., $\left.T_{1}\left(\lambda ; \lambda_{j}\right)\right|_{\lambda=\lambda_{j}} \psi_{j}=0, j=1,2$, we can calculate $a_{0}, b_{0}, c_{0}, d_{0}$. So we have the following theorem, 
Theorem 2.1 The onefold Darboux transformation with eigenvalue $\lambda_{1}$

$$
T_{1}\left(\lambda ; \lambda_{1}\right)=\left(\begin{array}{cc}
\lambda-\frac{1}{\Omega}\left|\begin{array}{cc}
\lambda_{1} \phi_{11} & \phi_{12} \\
\lambda_{2} \phi_{21} & \phi_{22}
\end{array}\right| & -\frac{1}{\Omega}\left|\begin{array}{cc}
\phi_{11} & \lambda_{1} \phi_{11} \\
\phi_{21} & \lambda_{2} \phi_{21}
\end{array}\right| \\
-\frac{1}{\Omega}\left|\begin{array}{cc}
\lambda_{1} \phi_{12} & \phi_{12} \\
\lambda_{2} \phi_{22} & \phi_{22}
\end{array}\right| & \lambda-\frac{1}{\Omega}\left|\begin{array}{cc}
\phi_{11} & \lambda_{1} \phi_{12} \\
\phi_{21} & \lambda_{2} \phi_{22}
\end{array}\right|
\end{array}\right),
$$

new solutions

$$
q^{[1]}=q-\frac{2}{\Omega}\left|\begin{array}{ll}
\phi_{11} & \lambda_{1} \phi_{11} \\
\phi_{21} & \lambda_{2} \phi_{21}
\end{array}\right|, \quad r^{[1]}=r-\frac{2}{\Omega}\left|\begin{array}{cc}
\lambda_{1} \phi_{12} & \phi_{12} \\
\lambda_{2} \phi_{22} & \phi_{22}
\end{array}\right|
$$

with

$$
\Omega=\left|\begin{array}{ll}
\phi_{11} & \phi_{12} \\
\phi_{21} & \phi_{22}
\end{array}\right|
$$

According to the form of $T_{1}$ in Eq.(8), the $n$-fold DT should be of the form

$$
T_{n}=T_{n}\left(\lambda ; \lambda_{1}, \lambda_{3}, \ldots, \lambda_{2 n-1}\right)=E \lambda^{n}+\sum_{i=0}^{n-1} P_{i} \lambda^{i}
$$

with

$$
E=\left(\begin{array}{ll}
1 & 0 \\
0 & 1
\end{array}\right), P_{i}=\left(\begin{array}{cc}
a_{i} & b_{i} \\
c_{i} & d_{i}
\end{array}\right)
$$

Theorem 2.2 If the function $q(x, t)$ is a solution of ecmKdV equation (2), then $q^{[n]}$ is new solution of (2) which defined by following,

$$
q^{[n]}=q+2 \frac{N_{2 n}}{W_{2 n}}
$$

and

with

$$
T_{n}=T_{n}\left(\lambda ; \lambda_{1}, \lambda_{2}, \ldots, \lambda_{2 n}\right)=\left(\begin{array}{ll}
\frac{\left(T_{2 n}\right)_{11}}{W_{2 n}} & \frac{\left(T_{2 n}\right)_{12}}{W_{2 n}} \\
\frac{\left(T_{2 n}\right)_{21}}{W_{2 n}} & \frac{\left(T_{2 n}\right)_{22}}{W_{2 n}}
\end{array}\right)
$$

$$
\begin{aligned}
& W_{2 n}=\left|\begin{array}{ccccccc}
\lambda_{1}^{n-1} \phi_{11} & \lambda_{1}^{n-1} \phi_{12} & \lambda_{1}^{n-2} \phi_{11} & \lambda_{1}^{n-2} \phi_{12} & \cdots & \phi_{11} & \phi_{12} \\
\lambda_{2}^{n-1} \phi_{21} & \lambda_{2}^{n-1} \phi_{22} & \lambda_{2}^{n-2} \phi_{21} & \lambda_{2}^{n-2} \phi_{22} & \cdots & \phi_{21} & \phi_{22} \\
\vdots & \vdots & \vdots & \vdots & \vdots & \vdots & \vdots \\
\lambda_{2 n}^{n-1} \phi_{2 n, 1} & \lambda_{2 n}^{n-1} \phi_{2 n, 2} & \lambda_{2 n}^{n-2} \phi_{2 n, 1} & \lambda_{2 n}^{n-2} \phi_{2 n, 2} & \cdots & \phi_{2 n, 1} & \phi_{2 n, 2}
\end{array}\right|, \\
& N_{2 n}=\left|\begin{array}{ccccccc}
\lambda_{1}^{n} \phi_{11} & \lambda_{1}^{n-1} \phi_{11} & \lambda_{1}^{n-2} \phi_{11} & \lambda_{1}^{n-2} \phi_{12} & \cdots & \phi_{11} & \phi_{12} \\
\lambda_{2}^{n} \phi_{21} & \lambda_{2}^{n-1} \phi_{21} & \lambda_{2}^{n-2} \phi_{21} & \lambda_{2}^{n-2} \phi_{22} & \cdots & \phi_{21} & \phi_{22} \\
\vdots & \vdots & \vdots & \vdots & \vdots & \vdots & \vdots \\
\lambda_{2 n}^{n} \phi_{2 n, 1} & \lambda_{2 n}^{n-1} \phi_{2 n, 1} & \lambda_{2 n}^{n-2} \phi_{2 n, 1} & \lambda_{2 n}^{n-2} \phi_{2 n, 2} & \cdots & \phi_{2 n, 1} & \phi_{2 n, 2}
\end{array}\right| \text {, } \\
& \left(T_{2 n}\right)_{11}=\left|\begin{array}{cccccccc}
\lambda^{n} & \lambda^{n-1} & 0 & \lambda^{n-2} & 0 & \cdots & 1 & 0 \\
\lambda_{1}^{n} \phi_{11} & \lambda_{1}^{n-1} \phi_{11} & \lambda_{1}^{n-1} \phi_{12} & \lambda_{1}^{n-2} \phi_{11} & \lambda_{1}^{n-2} \phi_{12} & \cdots & \phi_{11} & \phi_{12} \\
\lambda_{2}^{n} \phi_{21} & \lambda_{2}^{n-1} \phi_{21} & \lambda_{2}^{n-1} \phi_{22} & \lambda_{2}^{n-2} \phi_{21} & \lambda_{2}^{n-2} \phi_{22} & \cdots & \phi_{21} & \phi_{22} \\
\vdots & \vdots & \vdots & \vdots & \vdots & \vdots & \vdots & \vdots \\
\lambda_{2 n}^{n} \phi_{2 n, 1} & \lambda_{2 n}^{n-1} \phi_{2 n, 1} & \lambda_{2 n}^{n-1} \phi_{2 n, 2} & \lambda_{2 n}^{n-2} \phi_{2 n, 1} & \lambda_{2 n}^{n-2} \phi_{2 n, 2} & \cdots & \phi_{2 n, 1} & \phi_{2 n, 2}
\end{array}\right|, \\
& \left(T_{2 n}\right)_{12}=\left|\begin{array}{cccccccc}
0 & 0 & \lambda^{n-1} & 0 & \lambda_{n-2} & \cdots & 0 & 1 \\
\lambda_{1}^{n} \phi_{11} & \lambda_{1}^{n-1} \phi_{11} & \lambda_{1}^{n-1} \phi_{12} & \lambda_{1}^{n-2} \phi_{11} & \lambda_{1}^{n-2} \phi_{12} & \cdots & \phi_{11} & \phi_{12} \\
\lambda_{2}^{n} \phi_{21} & \lambda_{2}^{n-1} \phi_{21} & \lambda_{2}^{n-1} \phi_{22} & \lambda_{2}^{n-2} \phi_{21} & \lambda_{2}^{n-2} \phi_{22} & \cdots & \phi_{21} & \phi_{22} \\
\vdots & \vdots & \vdots & \vdots & \vdots & \vdots & \vdots & \vdots \\
\lambda_{2 n}^{n} \phi_{2 n, 1} & \lambda_{2 n}^{n-1} \phi_{2 n, 1} & \lambda_{2 n}^{n-1} \phi_{2 n, 2} & \lambda_{2 n}^{n-2} \phi_{2 n, 1} & \lambda_{2 n}^{n-2} \phi_{2 n, 2} & \cdots & \phi_{2 n, 1} & \phi_{2 n, 2}
\end{array}\right|,
\end{aligned}
$$




$$
\begin{aligned}
& \left(T_{2 n}\right)_{21}=\left|\begin{array}{cccccccc}
0 & \lambda^{n-1} & 0 & \lambda^{n-2} & 0 & \cdots & 1 & 0 \\
\lambda_{1}^{n} \phi_{12} & \lambda_{1}^{n-1} \phi_{11} & \lambda_{1}^{n-1} \phi_{12} & \lambda_{1}^{n-2} \phi_{11} & \lambda_{1}^{n-2} \phi_{12} & \cdots & \phi_{11} & \phi_{12} \\
\lambda_{2}^{n} \phi_{22} & \lambda_{2}^{n-1} \phi_{21} & \lambda_{2}^{n-1} \phi_{22} & \lambda_{2}^{n-2} \phi_{21} & \lambda_{2}^{n-2} \phi_{22} & \cdots & \phi_{21} & \phi_{22} \\
\vdots & \vdots & \vdots & \vdots & \vdots & \vdots & \vdots & \vdots \\
\lambda_{2 n}^{n} \phi_{2 n, 2} & \lambda_{2 n}^{n-1} \phi_{2 n, 1} & \lambda_{2 n}^{n-1} \phi_{2 n, 2} & \lambda_{2 n}^{n-2} \phi_{2 n, 1} & \lambda_{2 n}^{n-2} \phi_{2 n, 2} & \cdots & \phi_{2 n, 1} & \phi_{2 n, 2}
\end{array}\right|, \\
& \left(T_{2 n}\right)_{22}=\left|\begin{array}{cccccccc}
\lambda^{n} & 0 & \lambda^{n-1} & 0 & \lambda^{n-2} & \cdots & 0 & 1 \\
\lambda_{1}^{n} \phi_{12} & \lambda_{1}^{n-1} \phi_{11} & \lambda_{1}^{n-1} \phi_{12} & \lambda_{1}^{n-2} \phi_{11} & \lambda_{1}^{n-2} \phi_{12} & \cdots & \phi_{11} & \phi_{12} \\
\lambda_{2}^{n} \phi_{22} & \lambda_{2}^{n-1} \phi_{21} & \lambda_{2}^{n-1} \phi_{22} & \lambda_{2}^{n-2} \phi_{21} & \lambda_{2}^{n-2} \phi_{22} & \cdots & \phi_{21} & \phi_{22} \\
\vdots & \vdots & \vdots & \vdots & \vdots & \vdots & \vdots & \vdots \\
\lambda_{2 n}^{n} \phi_{2 n, 2} & \lambda_{2 n}^{n-1} \phi_{2 n, 1} & \lambda_{2 n}^{n-1} \phi_{2 n, 2} & \lambda_{2 n}^{n-2} \phi_{2 n, 1} & \lambda_{2 n}^{n-2} \phi_{2 n, 2} & \cdots & \phi_{2 n, 1} & \phi_{2 n, 2}
\end{array}\right| .
\end{aligned}
$$

Proof Combining Eq.(9) with the properties of Darboux transformation $\left.T_{n}\left(\lambda ; \lambda_{1}, \lambda_{2}, \ldots, \lambda_{2 n}\right)\right|_{\lambda=\lambda_{k}} \psi_{k}=$ $0, k=1,2, \ldots, 2 n$, the $P_{i}$ are solved by Cramer's rule. According to

$$
T_{x}+T M=M^{[n]} T, \quad T_{t}+T W=W^{[n]} T,
$$

and then comparing the coefficients of $\lambda^{n+1}$, it yields Eq.(10).

\section{Zero seed solution}

Taking the seed solution $q=r^{*}=0$, the spectral problem (4) reduces to

$$
\psi_{x}=M^{\prime} \psi, \quad \psi_{t}=W^{\prime} \psi,
$$

where

$$
M^{\prime}=\left(\begin{array}{cc}
-1 & 0 \\
0 & 1
\end{array}\right) \lambda, \quad W^{\prime}=\left(\begin{array}{cc}
-16 \beta & 0 \\
0 & 16 \beta
\end{array}\right) \lambda^{5}+\left(\begin{array}{cc}
-4 \alpha & 0 \\
0 & -4 \alpha
\end{array}\right) \lambda^{3} .
$$

By the simple calculation, the eigenfunctions corresponding to eigenvalue $\lambda_{2 j-1}$ are given by the following,

$$
\left(\begin{array}{l}
\phi_{2 j-1,1} \\
\phi_{2 j-1,2}
\end{array}\right)=\left(\begin{array}{c}
e^{-\lambda_{2 j-1} x+\left(-16 \beta \lambda_{2 j-1}^{5}+4 \alpha \lambda_{2 j-1}^{3}\right) t-\zeta} \\
e^{\lambda_{2 j-1} x+\left(16 \beta \lambda_{2 j-1}^{5}-4 \alpha \lambda_{2 j-1}^{3}\right) t+\zeta}
\end{array}\right), \quad j=1,2, \ldots, n,
$$

where $\zeta$ is a real constant.

\subsection{Soliton molecules}

In this subsection, we will consider soliton molecules of (2). Let $n=1, \zeta \neq 0$, and $\lambda_{1}=$ $a_{1}+i b_{1}$

$$
\left|q_{1-s}\right|^{2}=4 a_{1}^{2} \operatorname{sech}^{2}\left(2 a_{1}\left(H-\frac{\zeta}{a_{1}}\right)\right) .
$$

with

$$
H=-16 \beta t a_{1}{ }^{4}+160 \beta t a_{1}{ }^{2} b_{1}{ }^{2}-80 \beta t b_{1}{ }^{4}+4 \alpha t a_{1}{ }^{2}-12 \alpha t b_{1}{ }^{2}-x,
$$

It is easy to see that the interactions between $l$ solitons and $m$ molecules consisting of two same solitons when Eq.(10) satisfies the following resonance conditions from the expression of $H$,

$$
\lambda_{1}=-\lambda_{3}, \lambda_{5}=-\lambda_{7}, \ldots, \lambda_{4 m-3}=-\lambda_{4 m-1}, \quad n=2 m+l,
$$

The collision process of two soliton molecules consisting of two same solitons are shown in Figure 1 (b). To find a molecule consisting of $n$ solitons, the parameters in Eq.(10) are selected as follows:

$$
-16 \beta a_{2 j-1}^{4}+160 \beta a_{2 j-1}^{2} b_{2 j-1}^{2}+4 \alpha a_{2 j-1}^{2}-80 \beta b_{2 j-1}^{4}-12 \alpha b_{2 j-1}^{2}=v_{0},
$$


$\lambda_{1} \neq \lambda_{3} \ldots \neq \lambda_{2 n-1}, j=1,2, \ldots, n$. In addition, if we want to make the distance between two adjacent solitons in the molecule equal, then the constraint condition needs to add one more bases on Eq.(14):

$$
\frac{\zeta}{a_{2 j+1}}-\frac{\zeta}{a_{2 j-1}}=d_{0}
$$

where $v_{0}$ and $d_{0}$ are real constants. Figure $1(\mathrm{c})$ and $(\mathrm{d})$ show the molecule consisting of 3 solitons under condition (14) and (15). If we take $\alpha=1, \beta=0$, then the soliton molecules (12) reduced to the case of complex modified $\mathrm{KdV}$ equation as shown in [33].

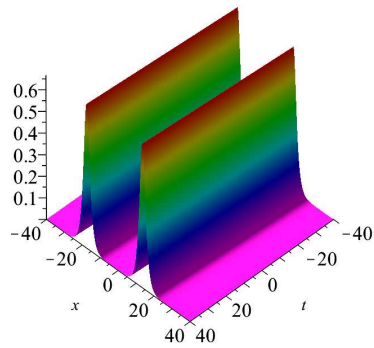

(a)

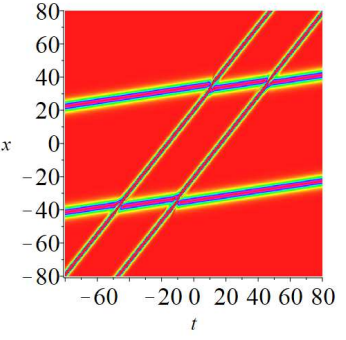

(b)

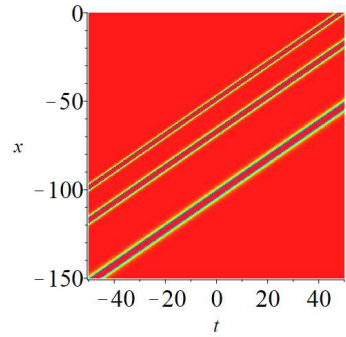

(c)

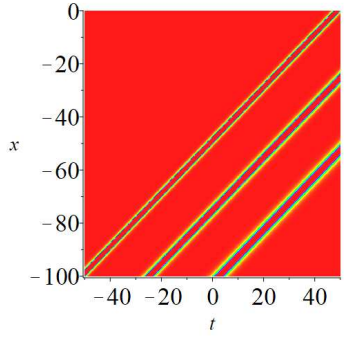

(d)

Figure 1: $\alpha=\beta=\frac{1}{2}$, (a) Soliton molecule consisting of two same solitons with $\lambda_{1}=-\lambda_{3}=-\frac{1}{3}+\frac{1}{3} i, \zeta=5$. (b) Elastic interaction property between two soliton molecules with $\lambda_{1}=-\lambda_{3}=\frac{1}{2}+\frac{\sqrt{6}}{6} i, \lambda_{5}=-\lambda_{7}=\frac{1}{3}+\frac{\sqrt{3}}{18} i, \zeta=10$. Soliton molecules consisting of three solitons with $\lambda_{1}=\frac{1}{2}+\frac{1}{2} i, \lambda_{5}=1+\frac{\sqrt{10}}{10} i, \zeta=50$. (c) $\lambda_{3}=\frac{3}{4}+\frac{\sqrt{195-20 \sqrt{71}}}{20} i$, (d) $\lambda_{3}=$ $\frac{2}{3}+\frac{\sqrt{1330-10 \sqrt{12209}}}{60} i$.

\subsection{Positon solutions}

In this subsection, we will construct the $n$-positon solutions of the ecmKdV equation. Taking seed solution $q=0$, it is trivial to see in Eq.(10) that $q^{[n]}$ becomes $\frac{0}{0}$ when $\lambda_{2 j-1} \rightarrow \lambda_{1}, i=$ $2,3, \ldots, n$. In general, we get a degenerate $n$-fold DT for the ecmKdV by setting $\lambda_{2 j-1}=\lambda_{1}+\epsilon$. Taking $\zeta=0$ and substituting $\psi_{j}$ difined by Eq.(11) into degenerate $n$-fold DT, we obtain smooth positon solutions of the ecmKdV using the higher order Taylor expansion with $\lambda_{2 j-1}=\lambda_{1}+\epsilon$.

Proposition 3.1 The $n$-fold DT in the degenerate limit $\lambda_{2 j-1} \rightarrow \lambda_{1}$ generates $(n-1)$-positon solution of the ecmKdV equation, which is given by

$$
q^{[n]}\left(x, t ; \lambda_{1}\right)=q+2 \frac{N_{2 n}^{\prime}}{W_{2 n}^{\prime}},
$$

with

$$
N_{2 n}^{\prime}=\left[\left.\frac{\partial^{n_{i}-1}}{\partial \epsilon^{n_{i}-1}}\right|_{\epsilon=0}\left(N_{2 n}\right)_{i j}\left(\lambda_{1}+\epsilon\right)\right], \quad W_{2 n}^{\prime}=\left[\left.\frac{\partial^{n_{i}-1}}{\partial \epsilon^{n_{i}-1}}\right|_{\epsilon=0}\left(W_{2 n}\right)_{i j}\left(\lambda_{1}+\epsilon\right)\right]
$$

Here $n_{i}=\left[\frac{i+1}{2}\right],[x]$ denotes the floor function of $x$ which is the function that takes as input a real number $x$, and gives as output the greatest integer less than or equal to $x$.

If we take $n=2$ in Proposition 3.1, one can obtain the explicit expression of 1-positon solution,

$$
q_{1-p}=-\frac{B_{1}}{B_{2}} a_{1} e^{-32 i \beta t b_{1}{ }^{5}+320 i \beta t a_{1}{ }^{2} b_{1}{ }^{3}-8 i \alpha t b_{1}{ }^{3}-160 i \beta t a_{1}{ }^{4} b_{1}+24 i \alpha t a_{1}{ }^{2} b_{1}-2 i x b_{1}}
$$


with

$$
\begin{aligned}
B_{1} & =\left(16+10240 i t b_{1}{ }^{3} a_{1}{ }^{2} \beta-10240 i \beta t a_{1}{ }^{4} b_{1}+768 i t b_{1} a_{1}{ }^{2} \alpha\right) \cosh \left(2 a_{1} H\right)+\left(2560 \beta t a_{1}{ }^{5}\right. \\
& \left.-15360 \beta t a_{1}{ }^{3} b_{1}{ }^{2}+2560 \beta t a_{1} b_{1}{ }^{4}-384 \alpha t a_{1}{ }^{3}+384 \alpha t a_{1} b_{1}{ }^{2}+32 x a_{1}\right) \sinh \left(2 a_{1} H\right), \\
B_{2} & =\left(102400 \beta^{2} a_{1}{ }^{10}+409600 \beta^{2} a_{1}{ }^{8} b_{1}{ }^{2}+614400 \beta^{2} a_{1}{ }^{6} b_{1}{ }^{4}+409600 \beta^{2} a_{1}{ }^{4} b_{1}{ }^{6}+102400 \beta^{2} a_{1}{ }^{2} b_{1}{ }^{8}\right. \\
& -30720 \alpha \beta a_{1}{ }^{8}-30720 \alpha \beta a_{1}{ }^{6} b_{1}{ }^{2}+30720 \alpha \beta a_{1}{ }^{4} b_{1}{ }^{4}+30720 \alpha \beta a_{1}{ }^{2} b_{1}{ }^{6}+2304 \alpha^{2} a_{1}{ }^{6} \\
& \left.+4608 \alpha^{2} a_{1}{ }^{4} b_{1}{ }^{2}+2304 \alpha^{2} a_{1}{ }^{2} b_{1}{ }^{4}\right) t^{2}+\left(2560 \beta x a_{1}{ }^{6}-15360 \beta x a_{1}{ }^{4} b_{1}{ }^{2}+2560 \beta x a_{1}{ }^{2} b_{1}{ }^{4}\right. \\
& \left.-384 \alpha x a_{1}{ }^{4}+384 \alpha x a_{1}{ }^{2} b_{1}{ }^{2}\right) t+16 x^{2} a_{1}{ }^{2}+2+2 \cosh \left(4 a_{1} H\right) .
\end{aligned}
$$

The 1-positon, 2-positon, and 3-positon solution are plotted in Figure 2.

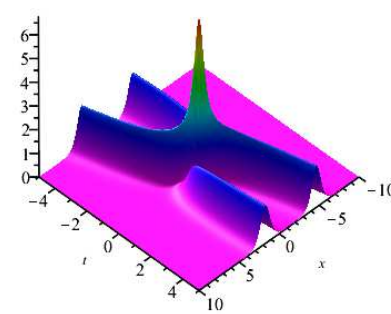

(a)

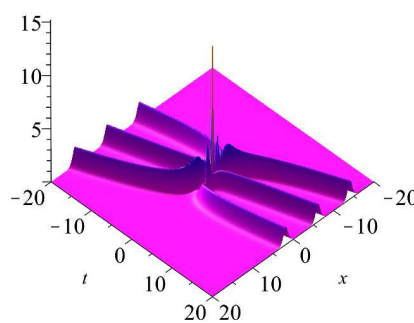

(b)

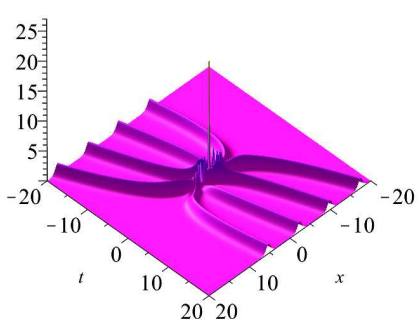

(c)

Figure 2: Positon solutions with $\alpha=\beta=0.5, a_{1}=0.65, b_{1}=0.1, \zeta=0$. From a to d are plots of 1-positon solution $\left|q_{1-p}\right|^{2}$, 2-positon solution $\left|q_{2-p}\right|^{2}$ and 3-positon solution $\left|q_{3-p}\right|^{2}$.

\section{Rational positon solutions}

In this section, we consider non-zero seed solution,

$$
\begin{aligned}
& q=c e^{i \rho} \\
& \rho=a x+b t \\
& b=\beta\left(a^{5}-20 a^{3} c^{2}+30 a c^{4}\right)+\alpha\left(a^{3}-6 a c^{2}\right),
\end{aligned}
$$

where $a, b, c$ are real constants. By using the principle of superposition of the linear differential equations, the new eigenfunctions corresponding to $\lambda_{j}$ can be rewritten as

$$
\psi_{j}=\left(\begin{array}{c}
\phi_{j 1} \\
\phi_{j 2}
\end{array}\right)=\left(\begin{array}{c}
z_{1} e^{Z_{1}}+z_{2} e^{Z_{2}}+z_{1}^{*} \frac{-2 \lambda_{j}-i\left(a+2 c_{1}\right)}{2 c} e^{Z_{2}}+z_{2}^{*} \frac{-2 \lambda_{j}-i\left(a-2 c_{1}\right)}{2 c} e^{Z_{1}} \\
-z_{1}^{*} e^{-Z_{1}}-z_{2}^{*} e^{-Z_{2}}+z_{1} \frac{2 \lambda_{j}+i\left(a+2 c_{1}\right)}{2 c} e^{-Z_{2}}+z_{2} \frac{2 \lambda_{j}+i\left(a-2 c_{1}\right)}{2 c} e^{-Z_{1}}
\end{array}\right)
$$

where

$$
\begin{gathered}
Z_{1}=i\left[\left(\frac{a}{2}+c_{1}\right) x+\left(\frac{b}{2}+2 c_{1} c_{2}\right) t\right], \quad Z_{2}=i\left[\left(\frac{a}{2}-c_{1}\right) x+\left(\frac{b}{2}-2 c_{1} c_{2}\right) t\right], \\
c_{1}=\frac{1}{2} \sqrt{a^{2}-4 \lambda_{j}^{2}+4 c^{2}-4 i a \lambda_{j}}, \\
c_{2}=\beta\left[8 \lambda_{j}^{4}-4 i a \lambda_{j}^{3}+\left(4 c^{2}-2 a^{2}\right) \lambda_{j}^{2}+i a \lambda_{j}\left(a^{2}-6 c^{2}\right)+\frac{1}{2} a^{4}-6 a^{2} c^{2}+3 c^{4}\right]+\alpha\left(\frac{a^{2}}{2}-c^{2}-2 \lambda_{j}^{2}+i a \lambda_{j}\right) .
\end{gathered}
$$

where $z_{1}, z_{2}$ are arbitrary complex constants. 
Proposition 4.1 Suppose that the eigenfunction obtained by the $n$-fold Darboux transformation degenerates at the eigenvalue $\lambda_{0}$, when $\lambda_{2 j-1} \rightarrow \lambda_{0}$, the degenerate $n$-fold Darboux transformation produces new solution

$$
q^{[n]}\left(x, t ; \lambda_{0}\right)=q+2 \frac{N_{2 n}^{\prime}}{W_{2 n}^{\prime}}
$$

where

$$
N_{2 n}^{\prime}=\left[\left.\frac{\partial^{n_{i}}}{\partial \epsilon^{n_{i}}}\right|_{\epsilon=0}\left(N_{2 n}\right)_{i j}\left(\lambda_{0}+\epsilon\right)\right], \quad W_{2 n}^{\prime}=\left[\left.\frac{\partial^{n_{i}}}{\partial \epsilon^{n_{i}}}\right|_{\epsilon=0}\left(W_{2 n}\right)_{i j}\left(\lambda_{0}+\epsilon\right)\right] .
$$

Here $n_{i}=\left[\frac{i+1}{2}\right] .[x]$ denotes the floor function.

When we set $z_{1}=c d_{1}, z_{2}=0$, the equation (17) can be rewritten as,

$$
\psi_{j}=\left(\begin{array}{c}
c d_{1} e^{i\left[\left(\frac{a}{2}+c_{1}\right) x+\left(\frac{b}{2}+2 c_{1} c_{2}\right) t\right]}-i d_{2}\left(\frac{a}{2}-i \lambda_{j}+c_{1}\right) e^{-i\left[\left(-\frac{a}{2}+c_{1}\right) x+\left(-\frac{b}{2}+2 c_{1} c_{2}\right) t\right]} \\
i d_{1}\left(\frac{a}{2}-i \lambda_{j}+c_{1}\right) e^{i\left[\left(-\frac{a}{2}+c_{1}\right) x+\left(-\frac{b}{2}+2 c_{1} c_{2}\right) t\right]}-c d_{2} e^{-i\left[\left(\frac{a}{2}+c_{1}\right) x+\left(\frac{b}{2}+2 c_{1} c_{2}\right) t\right]},
\end{array}\right)
$$

where

$$
d_{1}=e^{i c_{1}\left(s_{0}+s_{1} \epsilon+s_{2} \epsilon^{2}+\cdots+s_{n-1} \epsilon^{n-1}\right)}, \quad d_{2}=e^{-i c_{1}\left(s_{0}+s_{1} \epsilon+s_{2} \epsilon^{2}+\cdots+s_{n-1} \epsilon^{n-1}\right)}
$$

$s_{i} \in C(i=0,1,2, \ldots, n-1), a, b, c \in \mathbb{R}$. It is easy to verify that the eigenfunctions degenerate at $\lambda_{0}=c$, i.e., $\phi_{j}\left(\lambda_{0}\right)=0$ in the case of $a=0$. Combining the Proposition 4.1, we find that the rational positon solutions when we set $s_{i}=0, i=0,1, . ., n-1$,

$$
q_{1-r}=c-2 c \frac{L_{1}-1}{L_{1}+1}, \quad q_{2-r}=c+\frac{2 L_{2}}{L_{3}},
$$

The expressions of $L_{1}, L_{2}$ and $L_{3}$ are given in the Appendix A. Figure 3 shows the second-order rational positon solution $\left|q_{2-r}\right|$ and its density plot.

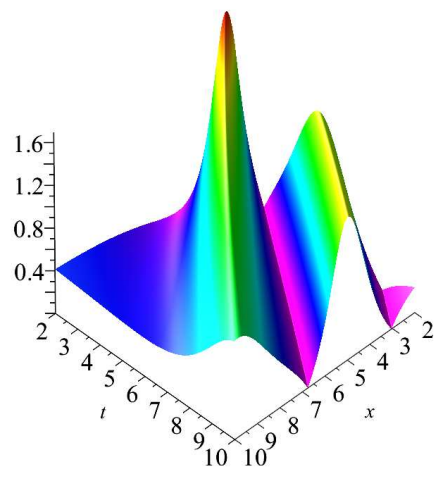

(a)

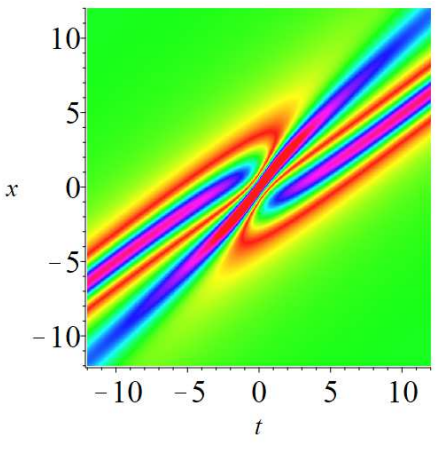

(b)

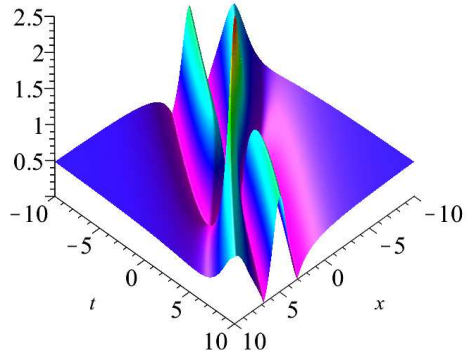

(c)

Figure 3: (a)(c) Second-order rational positon solution $\left|q_{2-r}\right|$ with $c=0.5, \alpha=0.8, \beta=0.2$. (b) The density plot of $\left|q_{2-r}\right|$.

\section{Rogue waves solution and their dynamic analysis}

In this section, starting with

$$
q=c e^{i \rho}, \quad \rho=a x+b t
$$

where

$$
b=\beta\left(a^{5}-20 a^{3} c^{2}+30 a c^{4}\right)+\alpha\left(a^{3}-6 a c^{2}\right), a, b, c \in \mathbb{R}, a, c \neq 0 .
$$


It can easily be verified that the eigenfunctions are defined in (19) degenerate at $\lambda_{0}=-\frac{i}{2} a+c$. Combining Proposition 4.1, we obtain the expression of the $n$-order RWs $q^{[n]}$. Due to the length and complexity of higher order RWs, we only give the explicit expression of the first-order RWs,

$$
q^{[1]}=c e^{i a\left[x+\left(a^{4} \beta-20 a^{2} c^{2} \beta+30 c^{4} \beta+a^{2} \alpha-6 \alpha c^{2}\right) t\right]} \frac{-A_{1}-160 i a^{3} \beta c^{2} t+480 i a \beta c^{4} t-48 i a \alpha c^{2} t+3}{A_{1}+1},
$$

where $A_{1}$ is given in Appendix B.

Through simple calculations, we find that $\left|q^{[1]}\right|^{2}=c^{2}$ when $x \rightarrow \infty, t \rightarrow \infty$ and $\left|q^{[1]}\right|^{2} \leq 9 c^{2}$. It is not difficult to find that the selection of parameters $d_{1}, d_{2}$ or equivalent to $s_{i}, i=0,1, \ldots, n-1$ will produce different types of RW. We assume $\alpha=\beta=0.5$ for the convenience of discussion. Next, Let's discuss the first-order to fifth-order RW because of the complexity of higher-order RW.

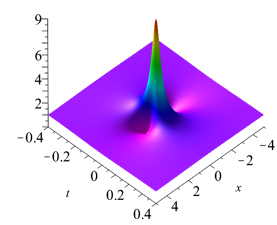

(a)

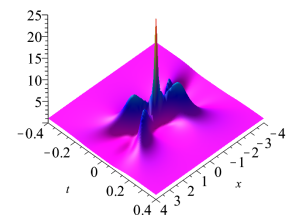

(b)

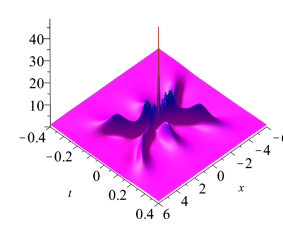

(c)

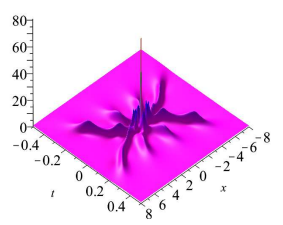

(d)

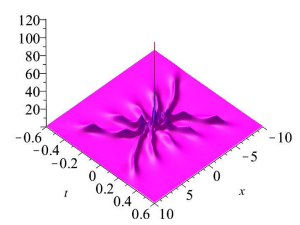

(e)

Figure 4: Fundamental patterns of the RW. $\alpha=\beta=0.5, c=1, s_{i}=0, i=0,1,2,3,4$. From (a) to (e) are firstorder RW $\left|q^{[1]}\right|^{2}$, second-order RW $\left|q^{[2]}\right|^{2}$, third-order RW $\left|q^{[3]}\right|^{2}$, fourth-order RW $\left|q^{[4]}\right|^{2}$, and fifth-order RW $\left|q^{[5]}\right|^{2}$ with $a=0.65,0.7,0.7,0.7,0.7$.

Setting $s_{i}=0, i=0,1, \ldots, n-1$, from the first-order RW $\left|q^{[1]}\right|^{2}$ to fifth-order RW $\left|q^{[5]}\right|^{2}$ in Figure 4 , it's clearly see that the $n$-order $\mathrm{RW}\left|q^{[n]}\right|^{2}$ takes the maximum value at $(x, t)=(0,0)$, and there are $n$ peaks on each side of $t=0(n>1)$. We call it the fundamental pattern of RW. Next, We analyze the contour line of the $\left|q^{[1]}\right|^{2}$ at different heights. First, Let's fix the value of $c$ and assume $c=1$,

1. At hight $c^{2}$, the expression of contour line is

$$
4 x^{2}+\left(20 a^{4} t-228 a^{2} t+96 t\right) x+25 a^{8} t^{2}-970 t^{2} a^{6}+5649 t^{2} a^{4}-5652 t^{2} a^{2}+576 t^{2}=1 .
$$

It's a hyperbola which has two asymptotes,

$$
x=\left(-\frac{\left(5 a^{4}-57 a^{2}+24\right)}{2} \pm\left(10 a^{3}-27 a\right)\right) t
$$

major axis $t=0$, imaginary axis: $l_{3}: x=-\frac{\left(5 a^{4}-57 a^{2}+24\right)}{2} t$.

2. At height $c^{2}+1, A_{2}=0$ which has two end points

$$
P_{1}=\left[\frac{\sqrt{7}}{2 a\left(10 a^{2}-27\right)},-\frac{\sqrt{7}\left(5 a^{4}-57 a^{2}+24\right)}{4 a\left(10 a^{2}-27\right)}\right], P_{2}=\left[-\frac{\sqrt{7}}{2 a\left(10 a^{2}-27\right)}, \frac{\sqrt{7}\left(5 a^{4}-57 a^{2}+24\right)}{4 a\left(10 a^{2}-27\right)}\right] .
$$

3. At height $\frac{c^{2}}{2}, A_{3}=0$, two centers of valleys $P_{3}=\left(0, \frac{\sqrt{3}}{2 c}\right), P_{4}=\left(0,-\frac{\sqrt{3}}{2 c}\right)$.

$A_{2}, A_{3}$ are defined in Appendix B. Figure 5 (a) gives the densityplots of $\left|q^{[1]}\right|^{2}$. Figure 5 (b), (c) and (d) show the contour of first-order RW $\left|q^{[1]}\right|^{2}$ with $h=c, c^{2}+1, \frac{c^{2}}{2}$. Similar to the idea of length and width defined in [14], the distance between $P_{1}$ and $P_{2}$ is length of first-order RW,

$$
\begin{aligned}
d_{L} & =\frac{\sqrt{7}}{a\left(10 a^{2}-27\right)} \sqrt{1+k_{l_{3}}^{2}} \\
& =\frac{\sqrt{7}}{2 a\left(10 a^{2}-27\right)} \sqrt{25 a^{8}-570 a^{6}+3489 a^{4}-2736 a^{2}+580},
\end{aligned}
$$




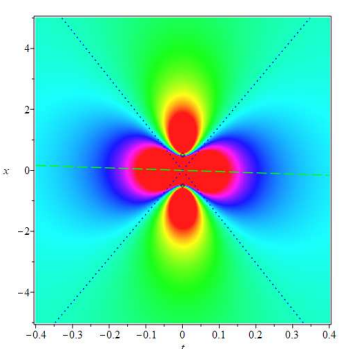

(a)

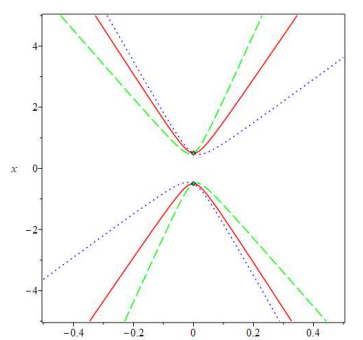

(b)

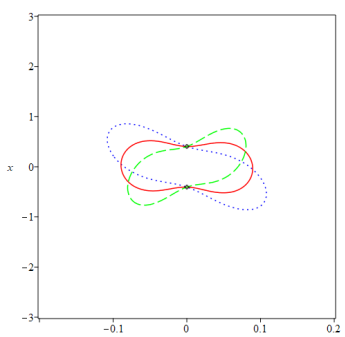

(c)

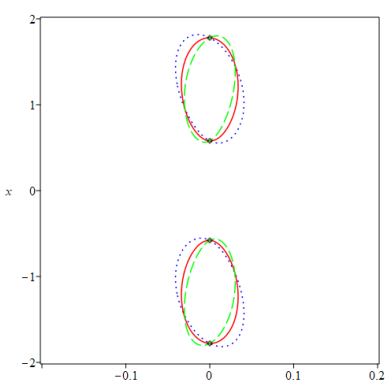

(d)

Figure 5: $\alpha=\beta=0.5, c=1$. (a)The density plot of the first-order RW $\left|q^{[1]}\right|^{2}$ with $a=0.65$. The blue point and green dashed line are the asymptote and imaginary axis of the contourline of $\left|q^{[1]}\right|^{2}$ at height $h=c^{2}$, From (b) to (d) are the contourline of first-order RW $\left|q^{[1]}\right|^{2}$ at height $h=c^{2}, c^{2}+1, \frac{c^{2}}{2} . a=0.5$ (blue point), $a=0.65$ (red curve), $a=0.8$ (green dotted line). (b) Two fixed points $(0,0.5),(0,-0.5)$, (c) two fixed points $(0,0.41),(0,-0.41)$, (d) four fixed points $(0,0.58)$, $(0,-0.58),(0,1.78),(0,-1.78)$.

the projection of line segment $P_{3} P_{4}$ in width direction is width of the first-order RW,

$$
d_{W}=\frac{\sqrt{3}}{\sqrt{1+k_{l_{3}}^{2}}}=\frac{2 \sqrt{3}}{\sqrt{25 a^{8}-570 a^{6}+3489 a^{4}-2736 a^{2}+580}} .
$$

Figure 6 (b) shows the length and width of the first-order RW when $c=1$. Combining Eq.(20) and Eq.(21), we can find that,

1. when $0<a<0.66$, the length keeps decreasing and the width keeps increasing;

2. when $0.66<a<1.64$, the length keeps increasing and the width keeps decreasing;

3. When $0.66<a<1.64$, the length keeps decreasing and the width keeps increasing;

4. When $a>1.64$, the length keeps increasing and the width keeps decreasing;

5. When $a<0$, the length and width plots are symmetrical to the $a>0$ plots.

This is the first effect of $a$. In addition, we find that the RW rotates counterclockwise with the increase of $a$ from the slope of the $l_{3}$. This is the second effect of $a$.

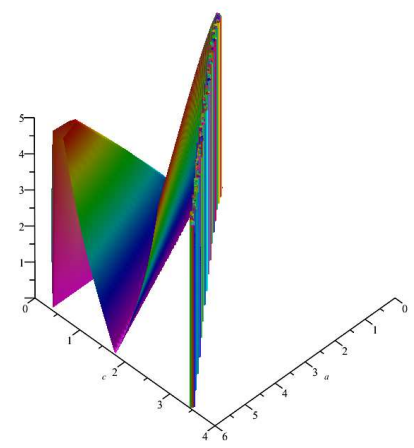

(a)

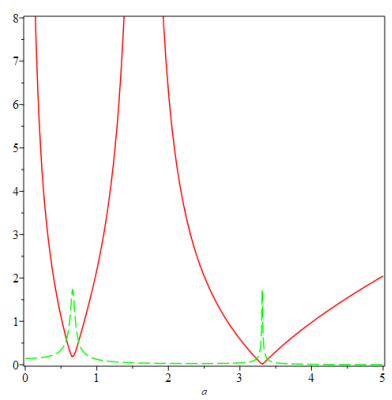

(b)

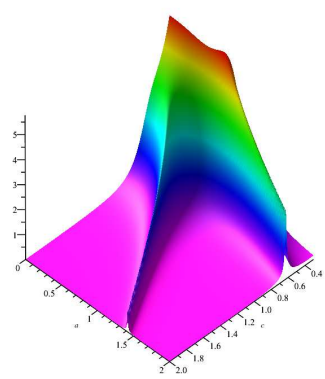

(c)

Figure 6: $\alpha=\beta=0.5$. The plots of first-order RW $\left|q^{[1]}\right|^{2}$ length (a) and width (c) at $h=c^{2}+1$. (b) The length(red curve) and width(green dashed line) of the first-order rogue waves $\left|q^{[1]}\right|^{2}$ at hight $c^{2}+1, c=1$, length and width get the extreme value when $a$ take $0.66,3.31$.

Continue the previous discussion and calculation ideas, when $c \neq 1$, the length and width of first-order RW,

$$
d_{c L}=\frac{\sqrt{8 c^{2}-1}}{2 a c^{2}\left(10 a^{2}-30 c^{2}+3\right)} \sqrt{4+\left(5 a^{4}-60 c^{2} a^{2}+30 c^{4}+3 a^{2}-6 c^{2}\right)^{2}}
$$




$$
d_{c W}=\frac{\sqrt{3}}{c \sqrt{1+k_{3 c}^{2}}}=\frac{2 \sqrt{3}}{c \sqrt{4+\left(5 a^{4}-60 c^{2} a^{2}+30 c^{4}+3 a^{2}-6 c^{2}\right)^{2}}} .
$$

Figure 6 (a) and (c) show the length and width of first-order RW under different parameters $a$ and $c$.

Taking $s_{1} \gg 1, n \geq 2, s_{i}=0, i=0,2,3 \ldots, n-1$, the RW will appear similar to the triangle structure, we call it the triangular patterns. Figure 7 shows triangular patterns of RW from second-order to fifth-order. Setting $s_{n-1} \gg 1, n \geq 3, s_{i}=0, i=0,1,2,3 \ldots, n-2$, the RW will have a ring-like structure, we call it the ring pattern. Figure 8 shows ring patterns of RW from third-order to fifth-order.

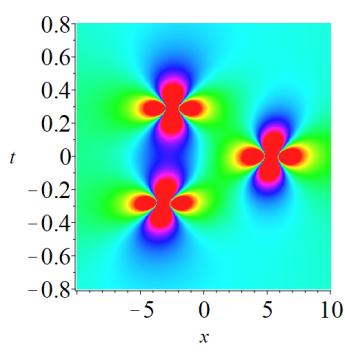

(a)

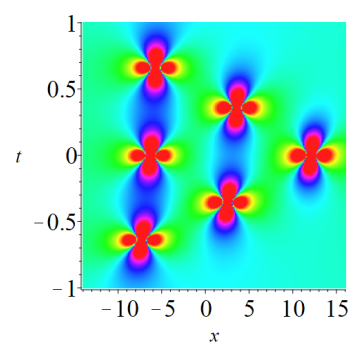

(b)

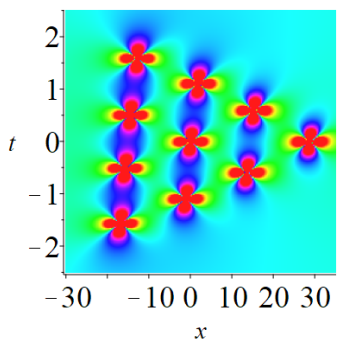

(c)

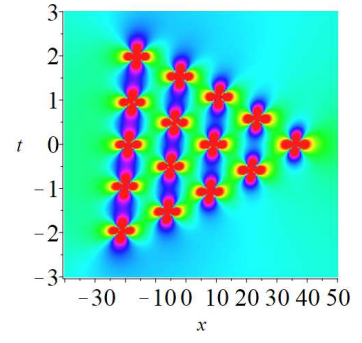

(d)

Figure 7: The triangular patterns of RW with $\alpha=\beta=0.5, c=1$. (a) The density plot of $\left|q^{[2]}\right|^{2}$ with $a=0.7, s_{0}=$ $0, s_{1}=100$. (b) The density plot of $\left|q^{[3]}\right|^{2}$ with $a=0.7, s_{0}=s_{2}=0, s_{1}=200$. (c) The density plot of $\left|q^{[4]}\right|^{2}$ with $a=0.7, s_{0}=s_{2}=s_{3}=0, s_{1}=1000$. (d) The density plot of $\left|q^{[5]}\right|^{2}$ with $a=0.7, s_{0}=s_{2}=s_{3}=s_{4}=0, s_{1}=1000$.

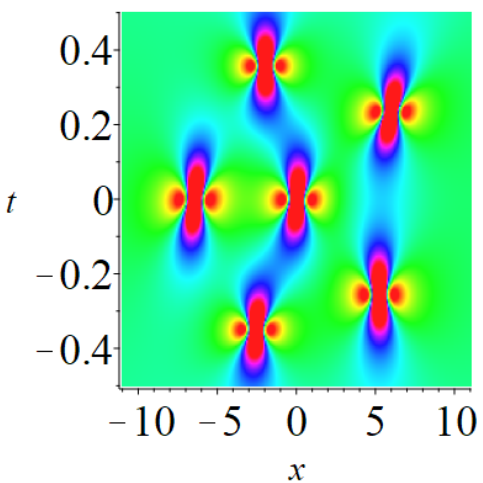

(a)

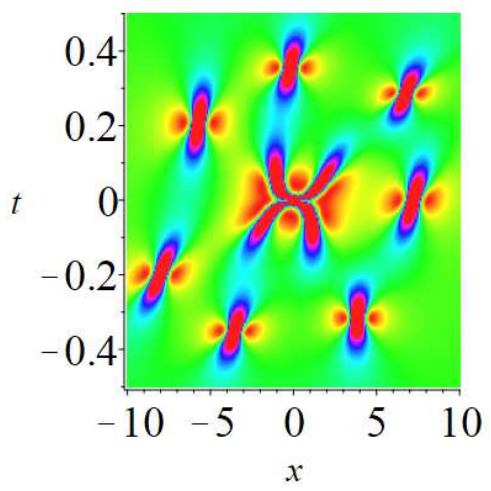

(b)

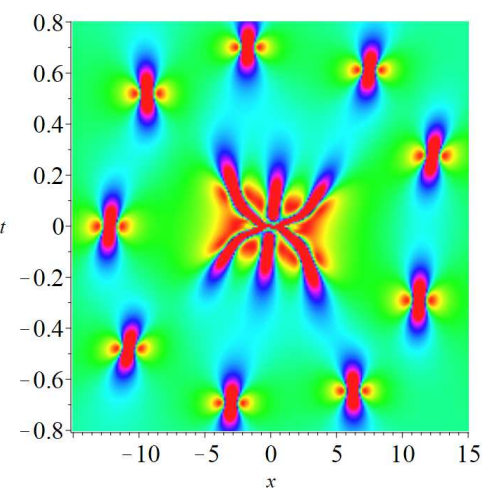

(c)

Figure 8: The ring patterns of RW with $\alpha=\beta=0.5, c=1$. (a) The density plot of $\left|q^{[3]}\right|^{2}$ with $a=0.7, s_{0}=s_{1}=$ $0, s_{2}=1000$. (b) The density plot of $\left|q^{[4]}\right|^{2}$ with $a=0.8, s_{0}=s_{1}=s_{2}=0, s_{3}=5000$. (c) The density plot of $\left|q^{[5]}\right|^{2}$ with $a=0.7, s_{0}=s_{1}=s_{2}=s_{3}, s_{4}=1000000$.

We can also get some combinations of triangular patterns and ring patterns. For example, from Figure 9(a)(b) we can see that a ring contains a triangle pattern. In Figure 9(c), a ring contains a ring pattern. The RW formed by the combination of the above three patterns can be called the standard decomposition of RW. Due to the diversity of the value of the parameter $s_{i}, i=0,1, \ldots, n-1$, we can of course obtain more than the above three patterns. Figure 10 shows non-standard decomposition of RW.

Remark 5.1 In the process of obtaining the fundamental patterns, triangular patterns and ring patterns above, we all choose $s_{0}=0$. If we set $s_{0} \neq 0$, We can also get similar above three patterns. Taking the third-order $R W\left|q^{[3]}\right|^{2}$ as an example, we can get the above three patterns with $s_{0}=10$ in Figure 11 . 


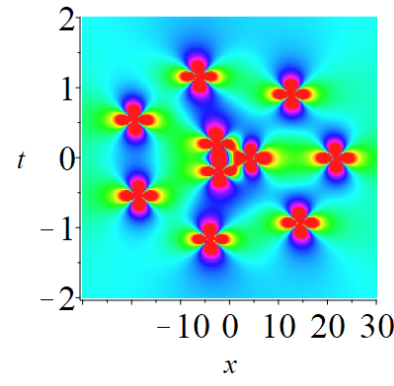

(a)

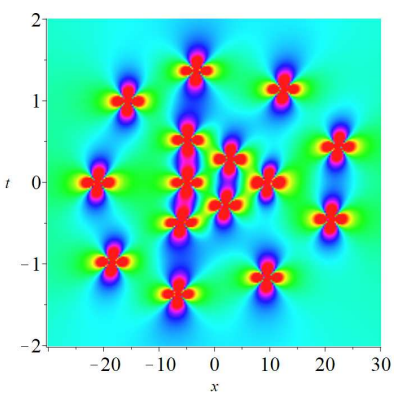

(b)

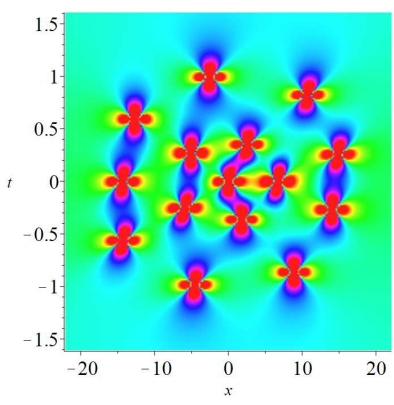

(c)

Figure 9: The standard decomposition of RW. $\alpha=\beta=0.5, a=0.7, c=1$. (a) The density plot of fourth-order RW $\left|q^{[4]}\right|^{2}$ with $s_{0}=s_{2}=0, s_{1}=50, s_{3}=10000000$. (b)The density plot of fifth-order RW $\left|q^{[5]}\right|^{2}$ with $s_{0}=s_{2}=s_{3}=0, s_{1}=$ $100, s_{4}=200000000$. (c) The density plot of fifth-order RW $\left|q^{[5]}\right|^{2}$ with $s_{0}=s_{1}=s_{2}=0, s_{3}=100000, s_{4}=10000000$.

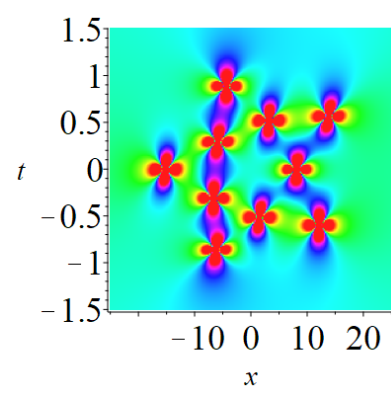

(a)

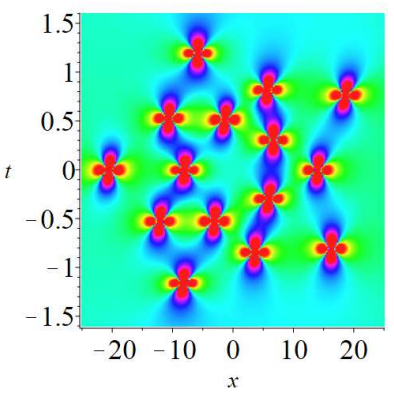

(b)

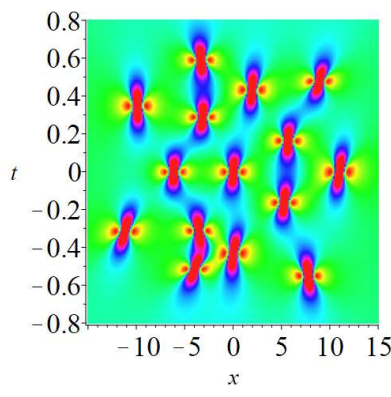

(c)

Figure 10: Non-standard decomposition of RW. $\alpha=\beta=0.5, a=0.7, c=1$. (a) The density plot of fourth-order RW $\left|q^{[4]}\right|^{2}$ with $s_{0}=s_{1}=s_{3}=0, s_{2}=10000$. (b) The density plot of fifth-order RW $\left|q^{[5]}\right|^{2}$ with $s_{0}=s_{1}=s_{3}=s_{4}=0, s_{2}=10000$. (c) The density plot of fifth-order RW $\left|q^{[5]}\right|^{2}$ with $s_{0}=s_{1}=s_{2}=s_{4}=0, s_{3}=10000$.

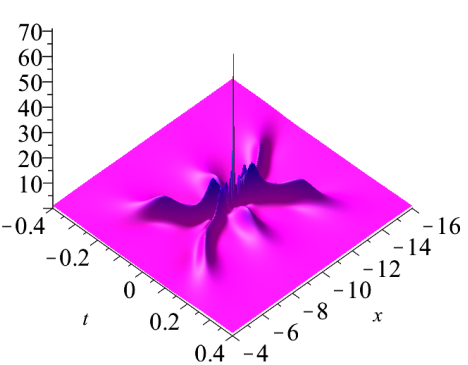

(a)

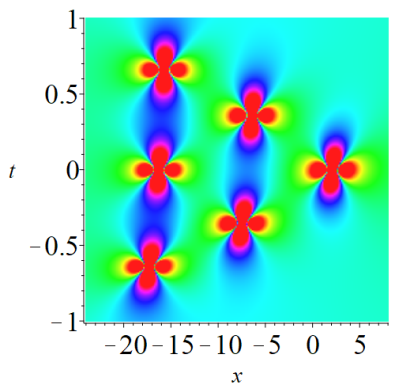

(b)

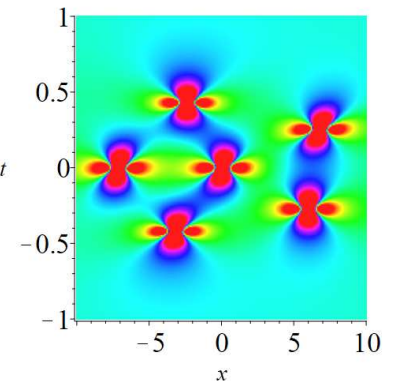

(c)

Figure 11: The RW similar to the fundamental pattern, triangular pattern and ring pattern when $s_{0} \neq 0 . \alpha=\beta=0.5, a=$ $0.7, c=1$. (a) The 3D plot of third-order RW $\left|q^{[3]}\right|^{2}$ with $s_{0}=10, s_{1}=0, s_{2}=0$. (b) The density plot of third-order RW $\left|q^{[3]}\right|^{2}$ with $s_{0}=10, s_{1}=200, s_{2}=0$. (c) The density plot of third-order RW $\left|q^{[3]}\right|^{2}$ with $s_{0}=10, s_{1}=0, s_{2}=2000$.

\section{Conclusions}

In this paper, we have presented the soliton molecules, positon solutions, rational positon solutions and rogue waves solutions for the extended complex modified KdV equation (2), and the plots of solutions are shown in the figures which are throughout the paper. If we consider the special case of the $\alpha=1, \beta=0$ or use $-\beta$ instead of $\beta$ and take the $q(x, t)$ is real function, our results can be reduce to the case of complex modified KdV equation which consider in $[14,31,33]$. 


\section{Acknowledgments}

Support is acknowledged from the National Science Foundation of China, Grant No.11901141.

\section{Data Availability Statement}

All data generated or analyzed during this study are included in this article.

\section{Conflict of interest}

The authors declare that they have no conflict of interest.

\section{Appendix A}

$$
\begin{aligned}
& L_{1}=3600 \beta^{2} c^{10} t^{2}-1440 \alpha \beta c^{8} t^{2}+144 \alpha^{2} c^{6} t^{2}+240 \beta c^{6} t x-48 \alpha c^{4} t x+4 c^{2} x^{2} \text {, } \\
& L_{2}=34560000 \beta^{4} t^{4} c^{21}-27648000 \alpha \beta^{3} t^{4} c^{19}+4608000 \beta^{3} t^{3} x c^{17}+8294400 \alpha^{2} \beta^{2} t^{4} c^{17} \\
& -1105920 \alpha^{3} \beta t^{4} c^{15}-2764800 \alpha \beta^{2} t^{3} x c^{15}+55296 \alpha^{4} t^{4} c^{13}+230400 \beta^{2} t^{2} x^{2} c^{13}+552960 \alpha^{2} \beta t^{3} x c^{13} \\
& -36864 \alpha^{3} t^{3} x c^{11}-92160 \alpha \beta t^{2} x^{2} c^{11}+364800 \beta^{2} t^{2} c^{11}+9216 \alpha^{2} t^{2} x^{2} c^{9}+5120 \beta t x^{3} c^{9}-8 c \\
& -115200 \alpha \beta t^{2} c^{9}-1024 \alpha t x^{3} c^{7}+8448 \alpha^{2} t^{2} c^{7}+14080 \beta t x c^{7}+\frac{128 x^{4} c^{5}}{3}-1792 \alpha t x c^{5}+64 x^{2} c^{3} \text {, } \\
& L_{3}=-20736000000 \beta^{6} t^{6} c^{30}+24883200000 \alpha \beta^{5} t^{6} c^{28}-12441600000 \alpha^{2} \beta^{4} t^{6} c^{26}-4147200000 \beta^{5} t^{5} x c^{26} \\
& +4147200000 \alpha \beta^{4} t^{5} x c^{24}+3317760000 \alpha^{3} \beta^{3} t^{6} c^{24}-1658880000 \alpha^{2} \beta^{3} t^{5} x c^{22}-345600000 \beta^{4} t^{4} x^{2} c^{22} \\
& -497664000 \alpha^{4} \beta^{2} t^{6} c^{22}+167040000 \beta^{4} t^{4} c^{20}+276480000 \alpha \beta^{3} t^{4} x^{2} c^{20}+331776000 \alpha^{3} \beta^{2} t^{5} x c^{20} \\
& +39813120 \alpha^{5} \beta t^{6} c^{20}-115200000 \alpha \beta^{3} t^{4} c^{18}-1327104 \alpha^{6} t^{6} c^{18}-82944000 \alpha^{2} \beta^{2} t^{4} x^{2} c^{18} \\
& -33177600 \alpha^{4} \beta t^{5} x c^{18}-15360000 \beta^{3} t^{3} x^{3} c^{18}+16128000 \beta^{3} t^{3} x c^{16}+29030400 \alpha^{2} \beta^{2} t^{4} c^{16} \\
& +9216000 \alpha \beta^{2} t^{3} x^{3} c^{16}+11059200 \alpha^{3} \beta t^{4} x^{2} c^{16}+1327104 \alpha^{5} t^{5} x c^{16}-3133440 \alpha^{3} \beta t^{4} c^{14} \\
& -7833600 \alpha \beta^{2} t^{3} x c^{14}-1843200 \alpha^{2} \beta t^{3} x^{3} c^{14}-384000 \beta^{2} t^{2} x^{4} c^{14}-552960 \alpha^{4} t^{4} x^{2} c^{14}+119808 \alpha^{4} t^{4} c^{12} \\
& +499200 \beta^{2} t^{2} x^{2} c^{12}+1198080 \alpha^{2} \beta t^{3} x c^{12}+153600 \alpha \beta t^{2} x^{4} c^{12}+122880 \alpha^{3} t^{3} x^{3} c^{12}-606400 \beta^{2} t^{2} c^{10} \\
& -55296 \alpha^{3} t^{3} x c^{10}-138240 \alpha \beta t^{2} x^{2} c^{10}-15360 \alpha^{2} t^{2} x^{4} c^{10}-5120 \beta t x^{5} c^{10}+145280 \alpha \beta t^{2} c^{8} \\
& +7680 \alpha^{2} t^{2} x^{2} c^{8}+\frac{12800 \beta t x^{3} c^{8}}{3}+1024 \alpha t x^{5} c^{8}-\frac{256 x^{6} c^{6}}{9}-8896 \alpha^{2} t^{2} c^{6}-8000 \beta t x c^{6}-\frac{512 \alpha t x^{3} c^{6}}{3} \\
& +1088 \alpha t x c^{4}-\frac{64 x^{4} c^{4}}{3}-48 x^{2} c^{2}-4 .
\end{aligned}
$$

\section{Appendix B}

$$
\begin{aligned}
A_{1} & =100 c^{2} a^{8} \beta^{2} t^{2}-800 t^{2} a^{6} \beta^{2} c^{4}+6000 t^{2} a^{4} \beta^{2} c^{6}+3600 c^{10} \beta^{2} t^{2}+120 c^{2} a^{6} \beta t^{2} \alpha-720 t^{2} a^{4} \beta c^{4} \alpha+720 t^{2} a^{2} \beta c^{6} \alpha \\
& -1440 c^{8} \beta t^{2} \alpha+36 c^{2} a^{4} \alpha^{2} t^{2}+144 c^{6} \alpha^{2} t^{2}+40 c^{2} a^{4} \beta t x+40 c^{2} a^{4} \beta t s_{0}-480 c^{4} a^{2} \beta t x-480 c^{4} a^{2} \beta t s_{0} \\
& +240 c^{6} \beta t x+240 c^{6} \beta t s_{0}+24 c^{2} a^{2} \alpha t x+24 c^{2} a^{2} \alpha t s_{0}-48 c^{4} \alpha t x-48 c^{4} \alpha t s_{0}+4 c^{2} x^{2}+8 c^{2} x s_{0}+4 c^{2} s_{0}{ }^{2} . \\
A_{2} & =16 x^{4}+\left(160 a^{4} t-1824 a^{2} t+768 t\right) x^{3}+\left(600 a^{8} t^{2}-10480 t^{2} a^{6}+66456 t^{2} a^{4}-42336 t^{2} a^{2}+13824 t^{2}\right. \\
& +40) x^{2}+\left(1000 a^{12} t^{3}-18200 a^{10} t^{3}+135480 a^{8} t^{3}-631464 a^{6} t^{3}+196128 a^{4} t^{3}+200 a^{4} t-228096 a^{2} t^{3}\right. \\
& \left.-2280 a^{2} t+110592 t^{3}+960 t\right) x+625 a^{16} t^{4}-8500 t^{4} a^{14}+95350 t^{4} a^{12}-442860 t^{4} a^{10}+1733841 t^{4} a^{8} \\
& +250 a^{8} t^{2}+282600 t^{4} a^{6}-8100 t^{2} a^{6}+1563408 t^{4} a^{4}+47850 t^{2} a^{4}+207360 t^{4} a^{2}-44856 t^{2} a^{2}+331776 t^{4} \\
& +5760 t^{2}-7 .
\end{aligned}
$$




$$
\begin{aligned}
A_{3} & =-8 x^{4}+\left(-80 a^{4}+912 a^{2}-384\right) t x^{3}+\left[28+\left(-300 a^{8}+5240 a^{6}-33228 a^{4}+21168 a^{2}-6912\right) t^{2}\right] x^{2} \\
& +\left[\left(-500 a^{12}+9100 a^{10}-67740 a^{8}+315732 a^{6}-98064 a^{4}+114048 a^{2}-55296\right) t^{3}+\left(140 a^{4}-1596 a^{2}\right.\right. \\
& +672) t] x+\left(-165888+4250 a^{14}-\frac{625}{2} a^{16}-103680 a^{2}-781704 a^{4}-141300 a^{6}-\frac{1733841}{2} a^{8}\right. \\
& \left.+221430 a^{10}-47675 a^{12}\right) t^{4}+\left(175 a^{8}-7590 a^{6}+43863 a^{4}-45396 a^{2}+4032\right) t^{2}-\frac{17}{2} .
\end{aligned}
$$

\section{References}

[1] Ablowitz, M.J., Clarkson, P.A.: Solitons, Nonlinear Evolution Equations and the Inverse Scattering Transformation. Cambridge University Press, Cambridge (1991)

[2] Ablowitz, M.J., Segur, H.: Solitons and the Inverse Scattering Transform. Society for Industrial and Applied Mathematics, Philadelphia (1981)

[3] Belokolos, E.D., Bobenko, A.I., Enolskij, V.Z., lts, A.R., Matveev, V.B.: AlgebroGeometrical Approach to Nonlinear Integrable Equations. Springer, Berlin (1994)

[4] Bluman, G.W., Cole, J.D.: Similarity Methods for Differential Equations. Springer, Berlin (1974)

[5] Erbay, H.A.: Nonlinear transverse waves in a generalized elastic solid and the complex modified Korteweg-de Vries Equation. Phys. Scr. 58, 9-14 (1998)

[6] Erbay, S., Suhubi, E.S.: Nonlinear wave propagation in micropolar media-II, Special cases, solitary waves and Painlevé analysis. Int. J. Eng. Sci. 27, 915-919 (1989)

[7] Guo, B.L., Ling, L.M., Liu, Q.P.: Nonlinear Schrödinger equation: generalized Darboux transformation and rogue wave solutions. Phys. Rev. E. 85, 026607 (2012)

[8] Gorbacheva, O.B., Ostrovsky, L.A.: Nonlinear vector waves in a mechanical model of a molecular chain. Physica D 8, 223-228 (1983)

[9] Guo, B.L., Tian, L.X., Yan, Z.Y., Ling, L.M., Wang Y.F.: Rogue Waves: Mathematical Theory and Applications in Physics. Zhejiang Science and Technology Press, Zhejiang (2017)

[10] Gu, C.H., Zhou, Z.X., Hu, H.S.: Darboux Transformations in Integrable Systems: Theory and Their Applications to Geometry. Springer, Berlin (2004)

[11] Hirota, R.: The Direct Method in Soliton Theory. Cambridge University Press, Cambridge (2004)

[12] He, J.S., Charalampidis, E.G., Kevrekidis, P.G., Frantzeskakis, D.J.: Rogue waves in nonlinear Schrödinger models with variable coefficients: application to Bose-Einstein condensates. Phys. Lett. A 378, 577-583 (2014)

[13] Herink, G., Kurtz, F., Jalali, B., Solli, D.R., Ropers, C.: Real-time spectral interferometry probes the internal dynamics of femtosecond soliton molecules. Science 356, 50-54 (2017)

[14] He, J.S., Wang, L.H., Li, L.J., Porsezian, K., Erdélyi, R.: Few-cycle optical rogue waves: Complex modified Korteweg-de Vries equation. Phys. Rev. E 89, 062917 (2014)

[15] Kharif, C., Pelinovsky, E., Slunyaev, A.: Rogue Waves in the Ocean. Springer, Berlin (2009)

[16] Karney, C.F.F., Sen, A., Chu, F.Y.F.: Nonlinear evolution of lower hybrid waves. Phys. Fluids 22, 940-952 (1979) 
[17] Liu, N.: Soliton and breather solutions for a fifth-order modified KdV equation with a nonzero background. Appl. Math. Lett. 104, 106256 (2020)

[18] Lou, S.Y.: Soliton molecules and asymmetric solitons in three fifth order systems via velocity resonance. J. Phys. Commun. 4, 041002 (2020)

[19] Liu, N., Guo, B.L.: Painlevé-type asymptotics of an extended modified KdV equation in transition regions. J. Differ. Equ. 280, 203-235 (2021)

[20] Liu, N., Guo, B.L., Wang, D.S., Wang, Y.F.: Long-time asymptotic behavior for an extended modified Korteweg-de Vries equation. Commun. Math. Sci. 17, 1877-1913 (2019)

[21] Lakomy, K., Nath, R., Santos, L.: Spontaneous crystallization and filamentation of solitons in dipolar condensates. Phys. Rev. A 85, 033618 (2012)

[22] Liu, W., Zhang, Y.S., He, J.S.: Dynamics of the smooth positons of the complex modified KdV equation. Waves Random Complex, 1-12 (2017)

[23] Miura, R.M.(Ed.): Bäcklund transformations, the inverse scattering method, solitons, and their applications: proceedings of the NSF Research Workshop on Contact Transformations, held in Nashville, Tennessee, 1974. Springer, Berlin (1976)

[24] Matveev, V.B., Salle, M.A.: Darboux Transformation and Solitons. Springer, Berlin (1991)

[25] Moslem, W.M., Sabry, R., El-Labany, S.K., Shukla, P.K.: Dust-acoustic rogue waves in a nonextensive plasma. Phys. Rev. E 84, 066402 (2011)

[26] Ren, B., Lin, J., Liu, P.: Soliton molecules and the CRE method in the extended mKdV equation. Commun. Theor. Phys. 75, 055005 (2020)

[27] Solli, D.R., Ropers, C., Koonath, P., Jalali, B.: Optical rogue waves. Nature 450, 1054-1057 (2007)

[28] Tao, Y.S., He, J.S.: Multisolitons, breathers, and rogue waves for the Hirota equation generated by the Darboux transformation. Phys. Rev. E 85, 026601 (2012)

[29] Walker, D.A.G., Taylor, P.H., Taylor, R.E.: The shape of large surface waves on the open sea and the Draupner New Year wave. Appl. Ocean Res. 26, 73-83 (2004)

[30] Wazwaz, A.M., Xu, G.Q.: An extended modified KdV equation and its Painlevé integrability. Nonlinear Dyn. 86, 1455-1460 (2016)

[31] Wang, X., Zhang, J.L., Wang, L.: Conservation laws, periodic and rational solutions for an extended modified Korteweg-de Vries equation. Nonlinear Dyn. 92, 1507-1506 (2018)

[32] Yan, Z.Y.: Vector financial rogue waves. Phys. Lett. A 375, 4274-4279 (2011)

[33] Zhang, Z., Yang, X.Y., Li, B.: Soliton molecules and novel smooth positons for the complex modified KdV equation. Appl. Math. Lett. 103, 106168 (2019) 


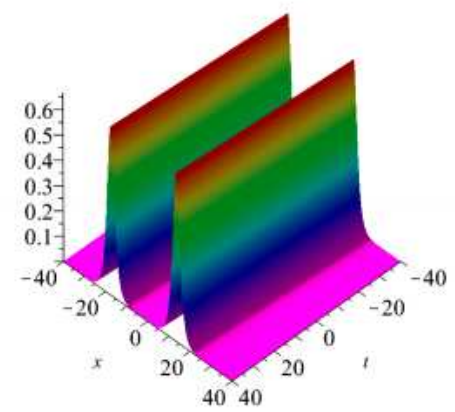

(a)

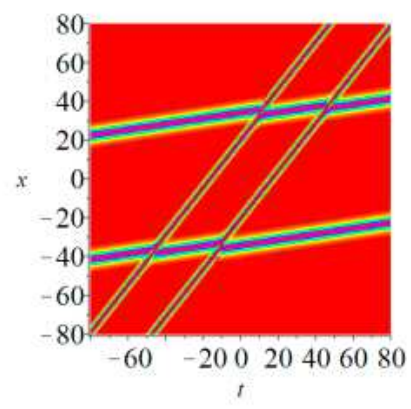

(b)

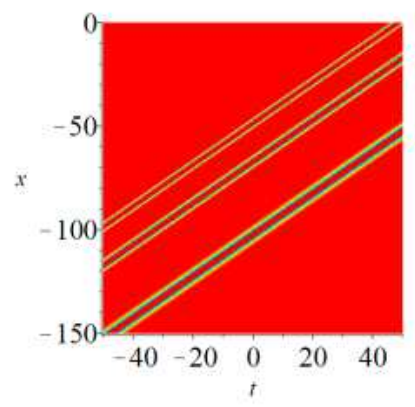

(c)

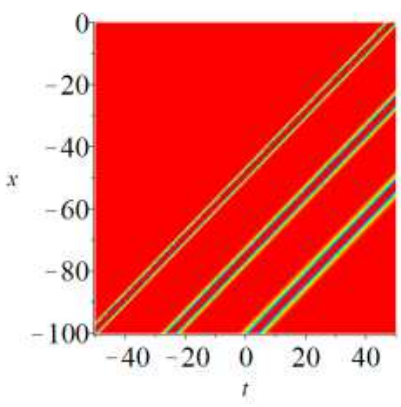

(d)

\section{Figure 1}

Please see the Manuscript PDF file for the complete figure caption

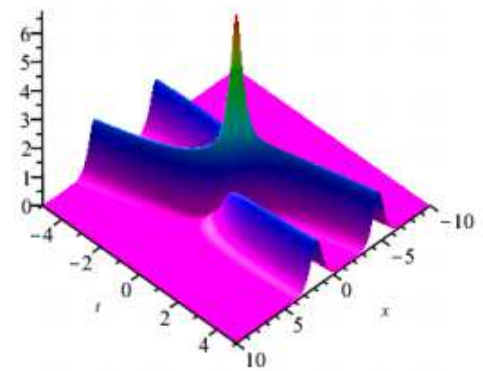

(a)

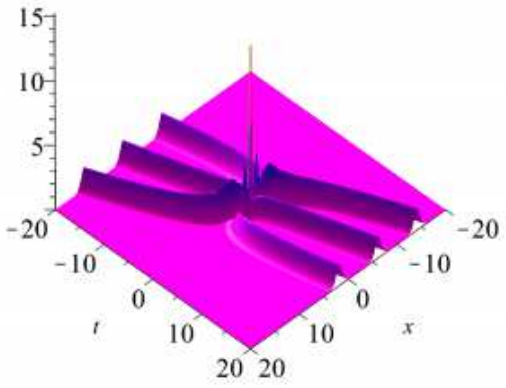

(b)

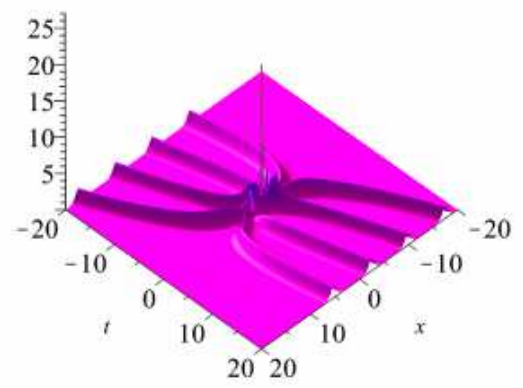

(c)

\section{Figure 2}

Please see the Manuscript PDF file for the complete figure caption 


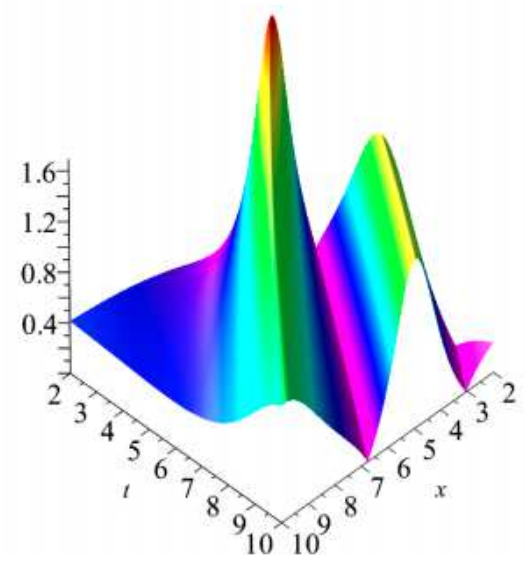

(a)

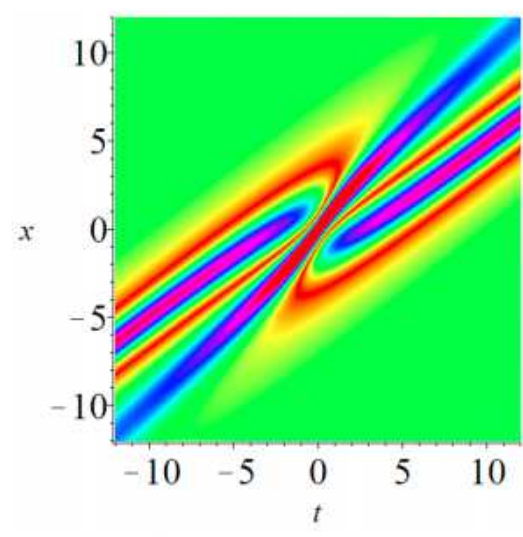

(b)

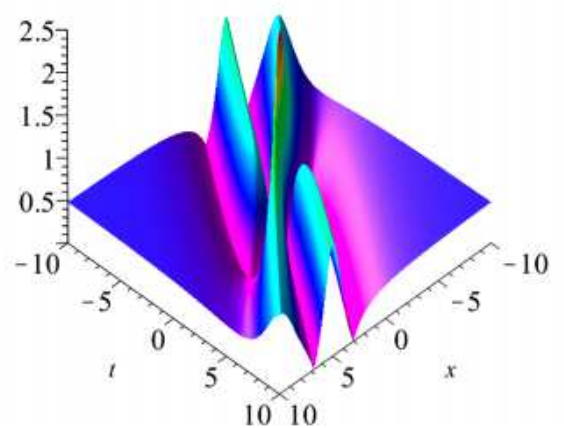

(c)

\section{Figure 3}

Please see the Manuscript PDF file for the complete figure caption

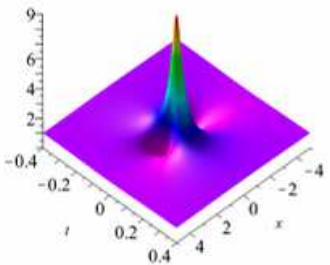

(a)

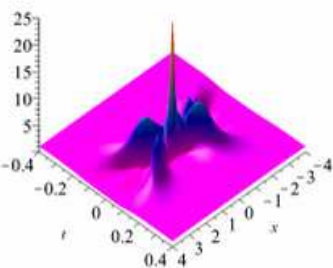

(b)

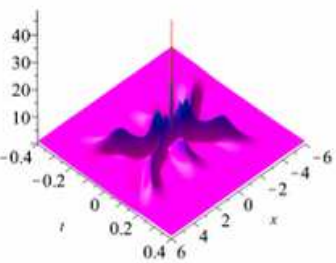

(c)

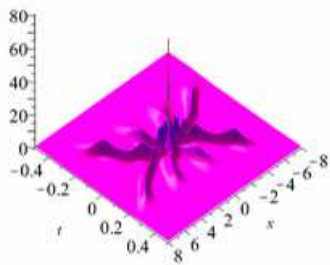

(d)

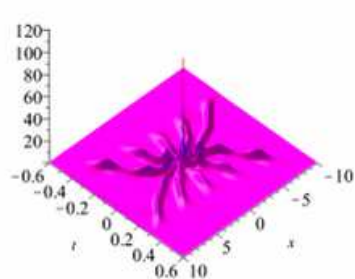

(e)

\section{Figure 4}

Please see the Manuscript PDF file for the complete figure caption

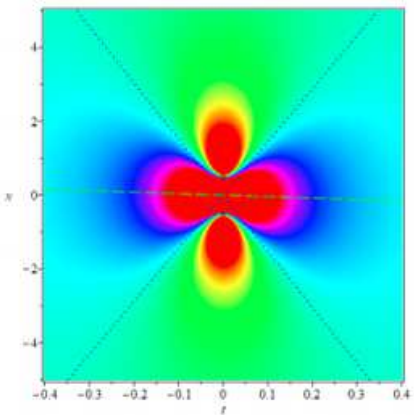

(a)

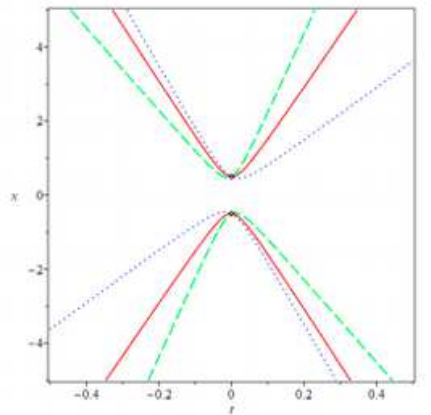

(b)

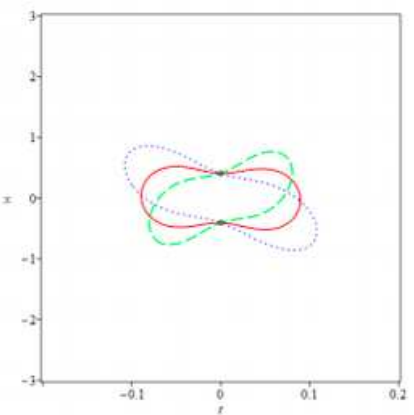

(c)

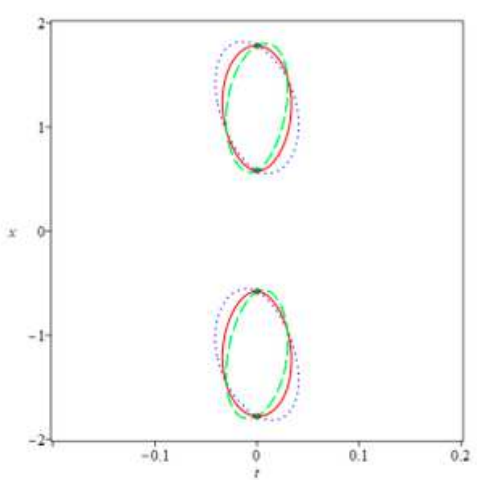

(d)

\section{Figure 5}

Please see the Manuscript PDF file for the complete figure caption 


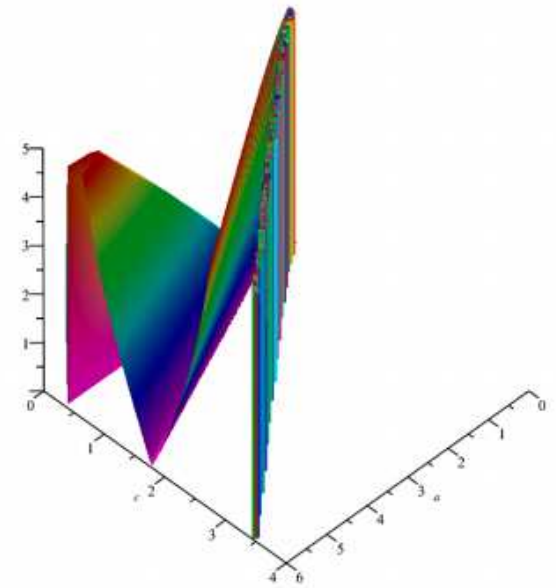

(a)

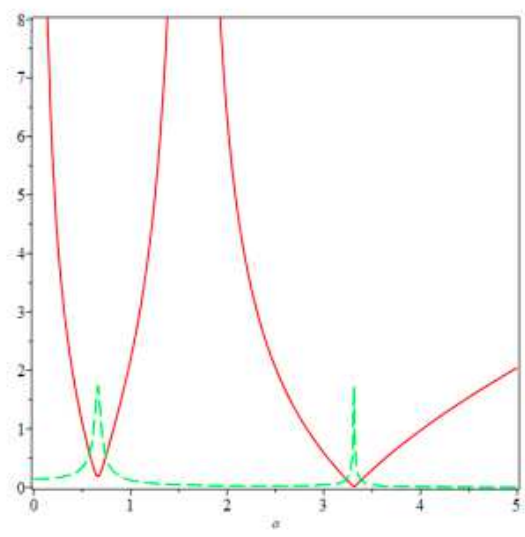

(b)

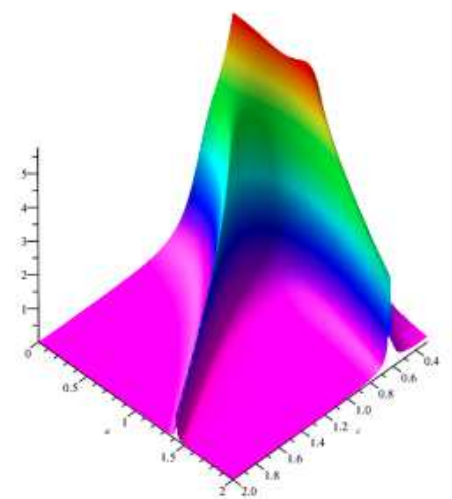

(c)

\section{Figure 6}

Please see the Manuscript PDF file for the complete figure caption

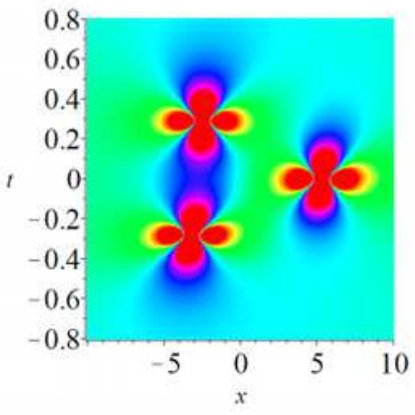

(a)

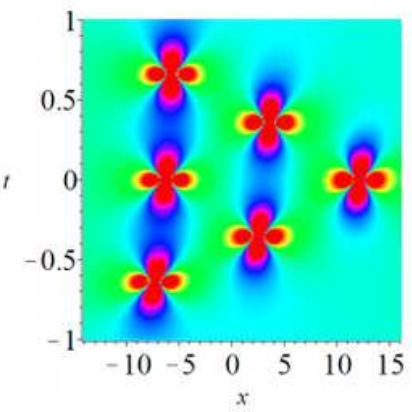

(b)

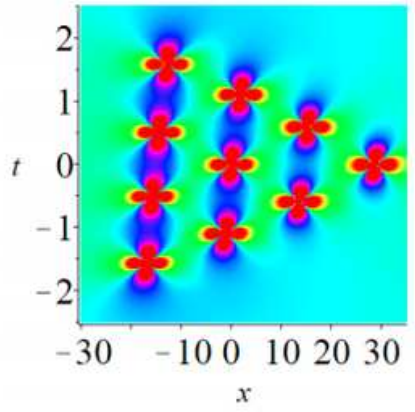

(c)

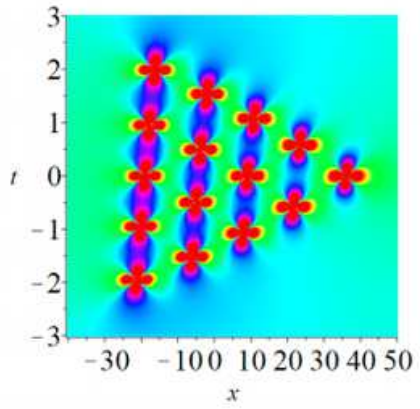

(d)

\section{Figure 7}

Please see the Manuscript PDF file for the complete figure caption 


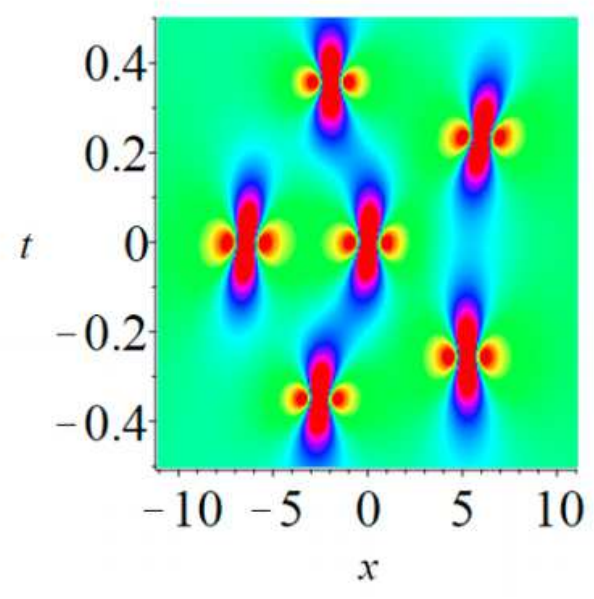

(a)

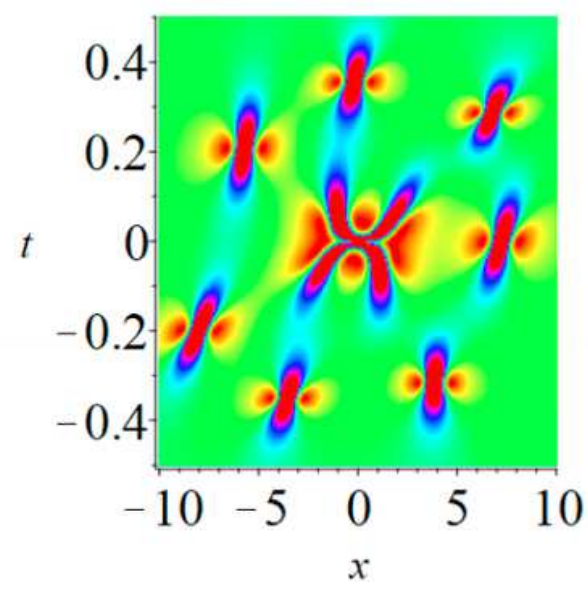

(b)

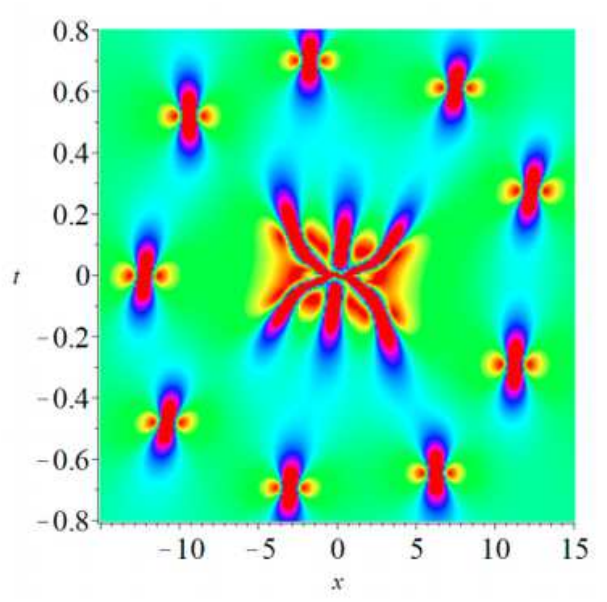

(c)

Figure 8

Please see the Manuscript PDF file for the complete figure caption

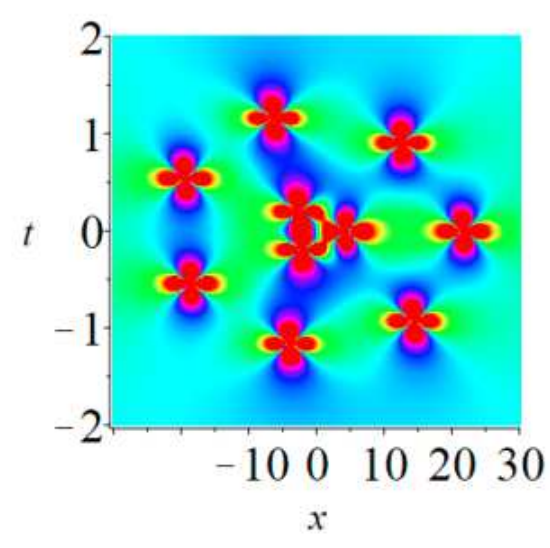

(a)

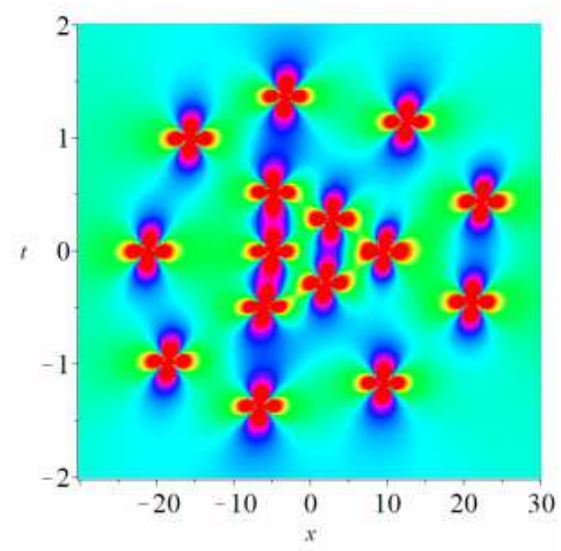

(b)

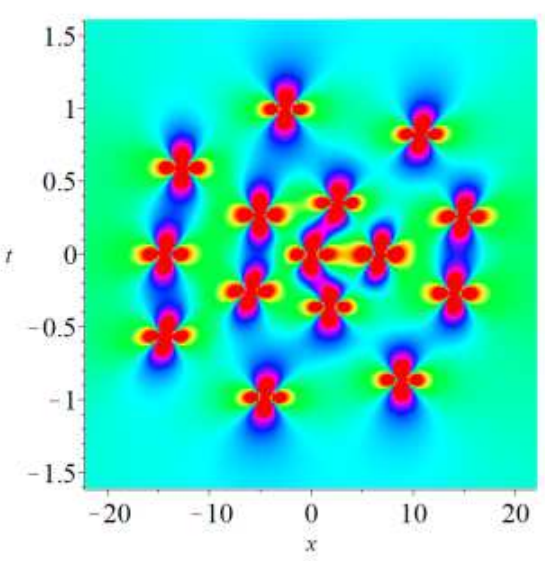

(c)

Figure 9

Please see the Manuscript PDF file for the complete figure caption 


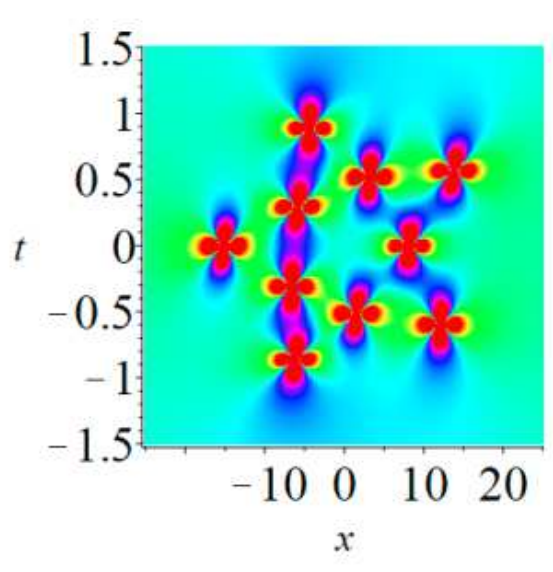

(a)

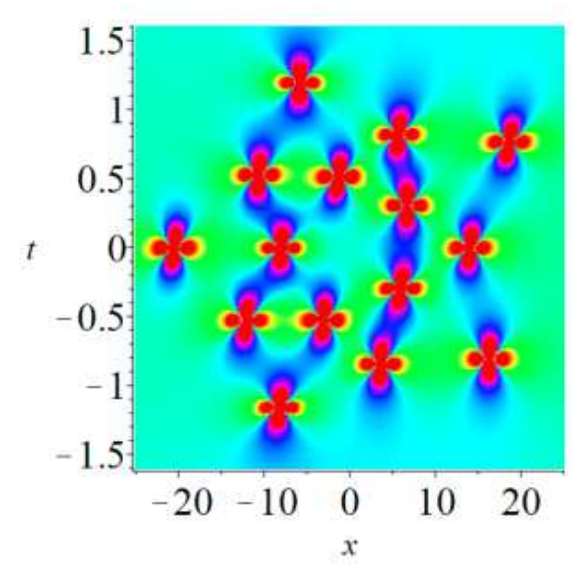

(b)

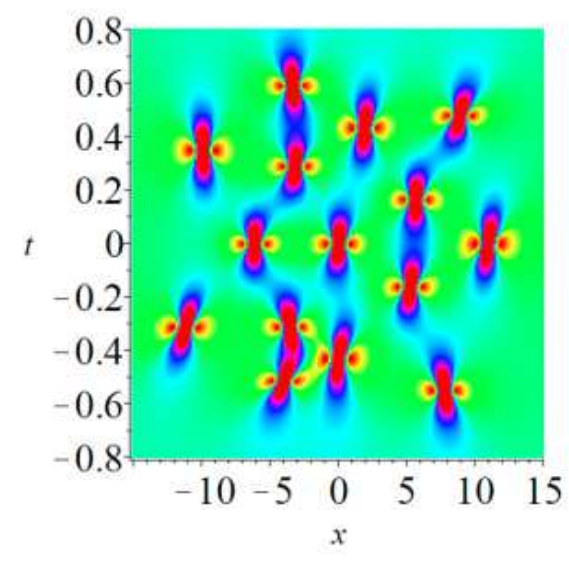

(c)

Figure 10

Please see the Manuscript PDF file for the complete figure caption

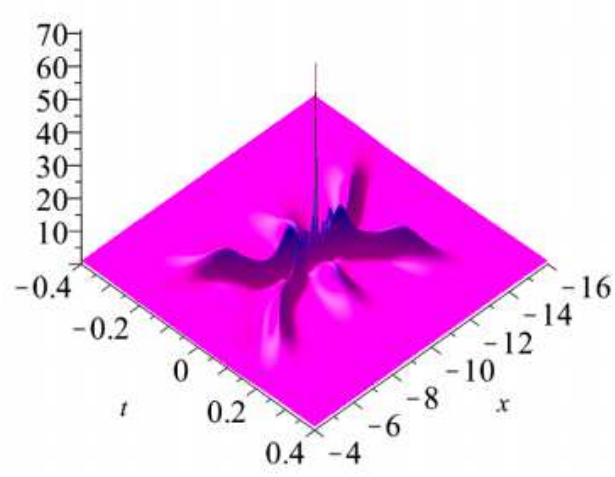

(a)

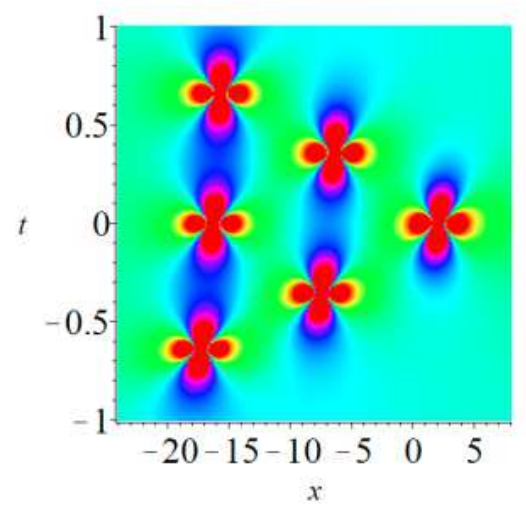

(b)

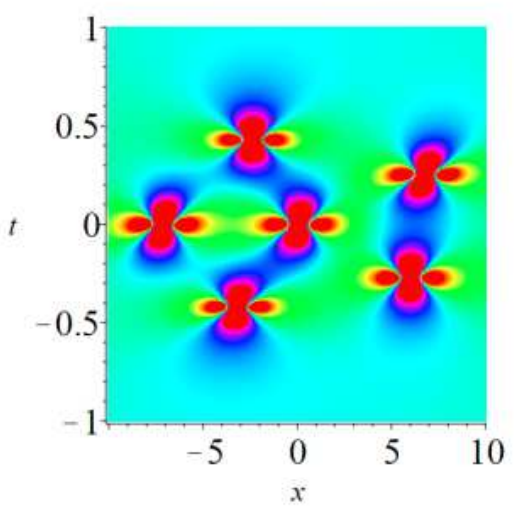

(c)

\section{Figure 11}

Please see the Manuscript PDF file for the complete figure caption 